\title{
Particle transport at arbitrary timescales with Poisson-distributed collisions
}

\author{
M. Baquero-Ruiz $\odot$, F. Manke, I. Furno $\odot$, A. Fasoli, and P. Ricci \\ École Polytechnique Fédérale de Lausanne (EPFL), Swiss Plasma Center (SPC), CH-1015 Lausanne, Switzerland
}

(Received 4 July 2019; published 22 November 2019)

\begin{abstract}
We develop a model to investigate the time evolution of the mean location and variance of a random walker subject to Poisson-distributed collisions at constant rate. The collisions are instantaneous velocity changes where a new value of velocity is generated from a model probability function. The walker is persistent, which means that it moves at constant velocity between collisions. We study three different cases of velocity transition functions and compute the transport properties from the evolution of the variance. We observe that transport can change character over time and that early times show features that, in general, depend on the initial conditions of the walker.
\end{abstract}

DOI: 10.1103/PhysRevE.100.052134

\section{INTRODUCTION}

Many results on random walks rely on the assumption of long timescales. This is particularly true in the case of continuous time random walks (CTRW), where the limit $t \rightarrow$ $\infty$ is widely used to simplify the Montrol-Weiss equation [1-3] and cast it in a form that ultimately leads to the diffusion equation or, more in general, to a type of fractional diffusion equation [1-4].

In some situations, however, one may be interested in the evolution of the walker at early times, i.e., when $t \lesssim \tau$, where $\tau$ is a typical collision time. In those cases, the results obtained for long timescales may not apply. This is exemplified by measurements of particle motion in a rarefied gas [5] or a liquid [6], the motion of organisms in biology $[7,8]$, and transport of particles in semiconductors $[9,10]$, where the walker dynamics between collisions plays an important role. This was already recognized in 1930 by Uhlenbeck and Ornstein in their model of Brownian motion [11,12], which allowed them to compute explicit results for all $t \geqslant 0$.

One simple model for dynamics between collisions is to assume that the walker moves with constant velocity. This persistent motion is a useful model in biology, where correlations at short timescales have been seen to be relevant to modeling of dispersal in biological systems [7]. It is also useful in physics, as it models the situation of no intercollision forces. The collisions are then understood to be instantaneous changes in velocity occurring randomly in time. In 1D persistent random walks (PRW) [9,13-15], for example, one usually describes the evolution of a walker moving at constant speed (modulus of velocity) but subject to random reversals of the direction of motion. The simplicity of PRW makes it amenable to analytical investigations that lead to time-changing transport properties and a close relationship to the Telegrapher's equation (TE) $[9,14]$.

In addition to the changes in direction, one can also consider changes in speed $[2,16]$. This has proven useful in studies of first-passage times of a persistent random walker [17] and, more recently, in some generalizations of the TE [18]. We pursue the idea by studying situations in which collisions lead to new (random) values of velocity arising from model probability density functions (PDFs). We study the time evolution of the variance of the walker position and show that the character of transport, understood here as the scaling of the variance as a function of time, changes over time. The results are valid for all times $t \geqslant 0$, in contrast to Refs. [2,16], where attention is paid to establishing asymptotic properties by assuming large spatial and temporal scales. In contrast to Ref. [19], where a Langevin approach is used, we develop a method that allows us to study different types of collisions and the effect of initial conditions of position and velocity.

We start with a theoretical framework (Sec. II). We develop a procedure to compute the time evolution of statistics of the walker's location (including the mean and variance) when subject to Poisson-distributed collisions. Then, we apply this method to three different types of velocity transition PDFs.

First we consider the case when, upon a collision, the walker transitions to a new random velocity independent of the precollision one (Sec. III). In this way we can model a tracer particle moving in a medium at constant temperature, a situation that may, for example, be of interest in studies of scattering of neutrons in a moderator [20] or scattering of electrons in a metal [10]. It can also find applications in studies of particle transport in plasmas [21], for example, in the context of suprathermal ions $[3,22,23]$, or in studies of particle dynamics in plasma turbulence [24]. We show that our approach yields exact expressions that can be compared to numerical simulations and give interesting insights of transport at short timescales. Notably, in certain circumstances, we observe superballistic behavior characterized by a $t^{3}$ scaling of the variance [19]. This constitutes an interesting analogy to a similar result observed in the formally different problem of relative separation of particles [24,25].

In Sec. IV we explore the case of addition of a random velocity. We show that this choice typically yields a $t^{3}$ scaling (at long enough $t$ ), providing a possible basis to the superballistic results observed in Sec. III.

In Sec. V, we study scaled velocity changes. This is the case when the walker velocity is multiplied by a constant 


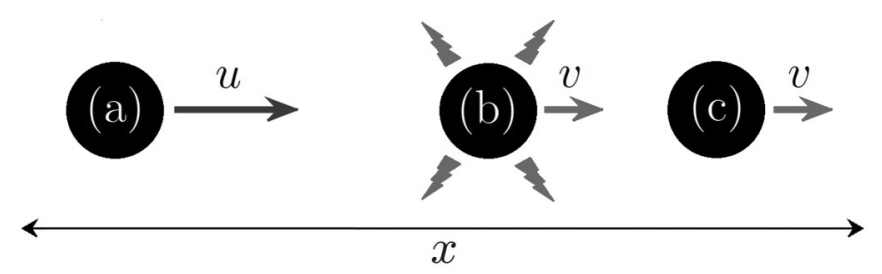

FIG. 1. Depiction of the system dynamics. A walker moves along $x$ with velocity $u$ (a) until a random event (a collision) makes the velocity change instantaneously to the new value $v(\mathrm{~b})$. The walker then moves with this velocity (c) until a new collision forces a change.

factor in each collision, a more artificial situation which nevertheless shares some similarities with exponential Fermi acceleration [26]. We show that transport can then have an exponential behavior. Also, we obtain a generalized version of the TE, thus showing that the formalism can recover results found in PRW while also enabling studies of other situations.

A summary of these results, as well as an outlook of future studies, is given in Sec. VI.

\section{THEORETICAL FRAMEWORK}

Let $p(x, t)$ be the PDF of the random 1D location $\chi(t)$ of a walker moving along " $x$ " at time " $t$ ". This means that $p(x, t) d x$ is the probability of finding the walker in the interval $(x, x+d x)$ at time $t$. The walker moves with constant velocity except at collisions, when the velocity transitions instantaneously to a new value (see Fig. 1). The resulting randomness in walker velocity leads us to consider $p(x, v, t)$, the PDF of walker location and velocity. For given $x, v$, and $t, p(x, v, t) d x d v$ is the probability of finding the walker with some velocity in $(v, v+d v)$ at the location $(x, x+d x)$ at time $t$. We note that

$$
p(x, t)=\int p(x, v, t) d v
$$

as $p(x, t)$ is just the PDF of location irrespective of velocity. Then, as shown in Appendix A, the evolution of $p(x, v, t)$ is determined by the transport equation [27]

$$
\left(\frac{\partial}{\partial t}+v \frac{\partial}{\partial x}+\gamma\right) p(x, v, t)=\gamma \int f(u, v) p(x, u, t) d u
$$

where $\gamma \equiv 1 / \tau$ is a constant collision rate (which for finite $\tau$ satisfies $\gamma>0)$ and $f(u, v)$ is a kernel giving the probability that, upon a collision, a walker moving with velocity $u$ transitions to a velocity $v$. The function $f$ needs to fulfill $\int f(u, v) d v=1$ for all $u$ (see Appendix A).

Equation (2) is a partial integrodifferential equation for $p(x, v, t)$, with known $\gamma$ and $f(u, v)$, which is in general difficult to solve. In transport studies, however, one is typically interested [2,15] in statistics of $\chi(t)$, such as the mean walker location $\langle\chi(t)\rangle=\int x p(x, t) d x \equiv \mu_{\chi}(t)$ and the mean square displacement $\left\langle\chi^{2}(t)\right\rangle=\int x^{2} p(x, t) d x \equiv$ $\sigma_{\chi}^{2}(t)+\left(\mu_{\chi}(t)\right)^{2}$, where $\sigma_{\chi}^{2}(t)$ is the variance. In other words, one wants to find moments [28] of $\chi(t)$. In that case, as discussed next, one does not need to find the full solution $p(x, v, t)$.
We assume that the walker moves in an unbounded space and that the Fourier transform of $p(x, v, t)$ on the spatial coordinate $x$ exists for $t \geqslant 0$ and all values of $v$. We state clearly the definition of the transform to avoid confusion with references that may use a different convention [29]:

$$
p(x, v, t) \stackrel{\mathcal{F}}{\rightarrow} p(\kappa, v, t) \equiv \int_{-\infty}^{\infty} p(x, v, t) e^{-\imath \kappa x} d x
$$

We use the transform variable $\kappa$, instead of $x$, to distinguish the transformed function from the original one. Similarly, we assume that the Laplace transform of $p(x, v, t)$ in $t$ exists for all $x$ and $v$ and the definition is

$$
p(x, v, t) \stackrel{\mathcal{L}}{\rightarrow} p(x, v, s) \equiv \int_{0}^{\infty} p(x, v, t) e^{-s t} d t .
$$

In this case, the transformed function is written as the original one but uses the transform variable $s$. The use of the Laplace transform in time is needed to include initial conditions at $t=$ 0 , which we assume to be of the form

$$
\left.p(x, v, t)\right|_{t=0}=g_{0}(x) f_{0}(v)
$$

In this expression $g_{0}(x)$ is the PDF of the (in general) random initial location $\chi_{0}$, and $f_{0}(v)$ is the PDF of the initial velocity $\mathcal{V}_{0}$. Then $\int g_{0}(x) d x=1$ and $\int f_{0}(v) d v=1$ so that $\left.\iint p(x, v, t)\right|_{t=0} d x d v=1$ (i.e., the probability of finding the walker anywhere moving at any speed when $t=0$ is one). Equation (4) means that the initial location and velocity are assumed to be statistically independent [28]. For what follows, we only consider the case where these initial PDFs have finite first and second moments and can be Fourier-transformed.

Taking Eq. (2) and applying a Fourier transform in $x$ followed by a Laplace transform in $t$ we obtain

$$
\begin{gathered}
(s+\imath \kappa v+\gamma) p(\kappa, v, s)-g_{0}(\kappa) f_{0}(v) \\
\quad=\gamma \int f(u, v) p(\kappa, u, s) d u
\end{gathered}
$$

where $g_{0}(\kappa)$ is the Fourier transform of $g_{0}(x)$. This form of the equation is very useful once one realizes that the Fourier transform of a PDF is its characteristic function [28]. Then, results similar to those used in probability theory [28] can be employed to compute the mean $\mu_{\chi}(t)$ and variance $\sigma_{\chi}^{2}(t)$ directly from $p(\kappa, t)$, without inverse-transforming to the original $x$-space [15]. For example,

$$
\begin{aligned}
\left.\frac{\partial}{\partial \kappa} p(\kappa, t)\right|_{\kappa=0} & =\left.\frac{\partial}{\partial \kappa}\left(\int_{-\infty}^{\infty} p(x, t) e^{-\imath \kappa x} d x\right)\right|_{\kappa=0} \\
& =\left.\int_{-\infty}^{\infty} \frac{\partial}{\partial \kappa}\left(p(x, t) e^{-\imath \kappa x}\right)\right|_{\kappa=0} d x \\
& =-\imath \int_{-\infty}^{\infty} x p(x, t) d x=-\imath \mu_{\chi}(t),
\end{aligned}
$$

and, following a similar procedure,

$$
\left.\frac{\partial^{2}}{\partial \kappa^{2}} p(\kappa, t)\right|_{\kappa=0}=-\sigma_{\chi}^{2}(t)-\left[\mu_{\chi}(t)\right]^{2} .
$$


In terms of $p(\kappa, s)$ we then have

$$
\begin{aligned}
& \mu_{\chi}(t)=\mathcal{L}^{-1}\left\{\left.l \frac{\partial}{\partial \kappa} p(\kappa, s)\right|_{\kappa=0}\right\} \\
& \sigma_{\chi}^{2}(t)=\mathcal{L}^{-1}\left\{-\left.\frac{\partial^{2}}{\partial \kappa^{2}} p(\kappa, s)\right|_{\kappa=0}\right\}-\left[\mu_{\chi}(t)\right]^{2} .
\end{aligned}
$$

In the next sections we show that Eqs. (7) together with Eq. (5) can be used to find exact analytic expressions for $\mu_{\chi}(t)$ and $\sigma_{\chi}^{2}(t)$. Higher-order moments of $p(x, t)$ can be computed in a similar fashion.

\section{RANDOM VELOCITY UPON A COLLISION}

\section{A. Model}

As a first example of the applicability of the theory of Sec. II, we consider

$$
f(u, v)=f_{R}(v),
$$

where $f_{R}$ is a PDF with finite first- and second-order moments. This is the case when, upon a collision, the walker transitions to a new random velocity $\mathcal{V}$ distributed $f_{R}(v)$, and the new velocity is independent of the one prior to the collision. This choice can, for example, model situations where a tracer particle moves through a medium at constant temperature. In that case, collisions make the particle jump to random velocities allowed by a velocity distribution associated to the medium temperature.

Since $\quad \int f_{R}(v) p(\kappa, u, s) d u=f_{R}(v) \int p(\kappa, u, s) d u=$ $f_{R}(v) p(\kappa, s)$ [see Eq. (1)], replacing $f_{R}$ in Eq. (5) gives

$$
(s+\imath \kappa v+\gamma) p(\kappa, v, s)-g_{0}(\kappa) f_{0}(v)=\gamma f_{R}(v) p(\kappa, s),
$$

which leads to

$$
p(\kappa, v, s)=\frac{g_{0}(\kappa) f_{0}(v)}{s+\imath \kappa v+\gamma}+\frac{\gamma f_{R}(v)}{s+\imath \kappa v+\gamma} p(\kappa, s) .
$$

Then, integrating on both sides with respect to $v$, the left-hand side (LHS) becomes $\int p(\kappa, v, s) d v=p(\kappa, s)$, which allows us to solve for $p(\kappa, s)$ and obtain

$$
\begin{aligned}
& p(\kappa, s) \\
& \quad=\left[\int \frac{g_{0}(\kappa) f_{0}(v)}{s+\imath \kappa v+\gamma} d v\right]\left[1-\gamma \int \frac{f_{R}(v)}{s+\imath \kappa v+\gamma} d v\right]^{-1} .
\end{aligned}
$$

This expression gives the complete time evolution of $p(x, t)$ in the transformed spaces.

Since $\quad p(\kappa, s)=\mathcal{L}\{\mathcal{F}\{p(x, t)\}\}=\mathcal{L}\left\{\int_{-\infty}^{\infty} p(x, t) e^{\imath \kappa x} d x\right\}$, we have $\int_{-\infty}^{\infty} p(x, t) d x=\mathcal{L}^{-1}\left\{\left.p(\kappa, s)\right|_{\kappa=0}\right\}$. Evaluating the expression in Eq. (9) at $\kappa=0$, we have

$$
\begin{aligned}
\left.p(\kappa, s)\right|_{\kappa=0} & =\left[\int \frac{f_{0}(v)}{s+\gamma} d v\right]\left[1-\gamma \int \frac{f_{R}(v)}{s+\gamma} d v\right]^{-1} \\
& =\left(\frac{1}{s+\gamma}\right)\left(1-\gamma \frac{1}{s+\gamma}\right)^{-1}=\frac{1}{s}
\end{aligned}
$$

since $\left.g_{0}(\kappa)\right|_{\kappa=0}=1, \int f_{0}(v) d v=1$, and $\int f_{R}(v) d v=1$. Therefore, $\int_{-\infty}^{\infty} p(x, t) d x=\mathcal{L}^{-1}\left\{\frac{1}{s}\right\}=1$ as expected, since probability needs to be conserved at all times $t \geqslant 0$.

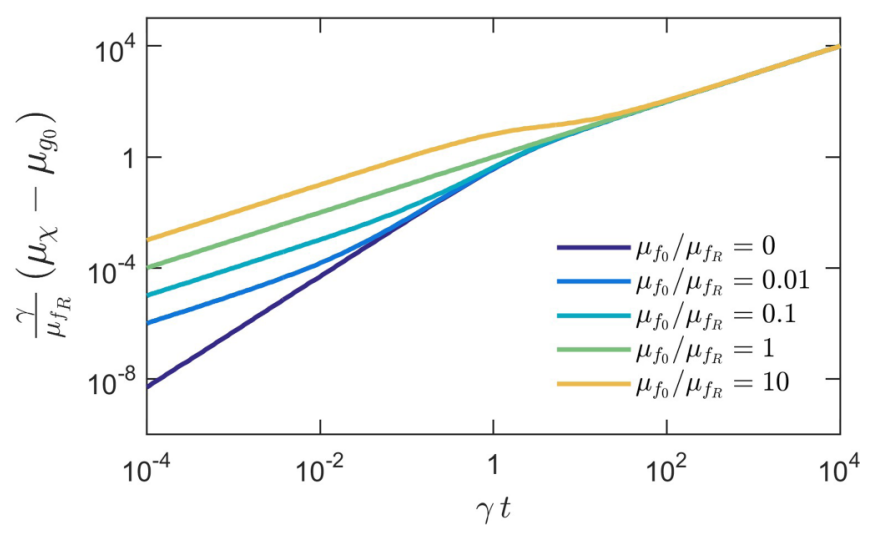

FIG. 2. Evolution of $\mu_{\chi}(t)$ for different ratios $\mu_{f_{0}} / \mu_{f_{R}}$ of mean initial and mean post-collision velocities. We assume here $\mu_{f_{R}}>0$. As Eq. (10) shows, the functional form of $\mu_{\chi}(t)$ only depends on $\mu_{f_{0}} / \mu_{f_{R}}$ and $\gamma t$ (the mean number of collisions at time $t$ ), once $\mu_{g_{0}}$ has been subtracted.

Inserting Eq. (9) into Eqs. (7) allows us to compute the mean walker location (see Appendix B):

$$
\begin{aligned}
\mu_{\chi}(t) & =\mathcal{L}^{-1}\left\{\frac{\mu_{g_{0}}}{s}+\frac{\mu_{f_{0}}}{s(s+\gamma)}+\frac{\gamma \mu_{f_{R}}}{s^{2}(s+\gamma)}\right\} \\
& =\mu_{g_{0}}+\frac{1}{\gamma}\left[\mu_{f_{0}}\left(1-e^{-\gamma t}\right)+\mu_{f_{R}}\left(-1+\gamma t+e^{-\gamma t}\right)\right] .
\end{aligned}
$$

Here, $\mu_{g_{0}}$ and $\mu_{f_{0}}$ are the means of the initial conditions $\chi_{0}$ and $\mathcal{V}_{0}$ with PDFs $g_{0}$ and $f_{0}$, respectively, and $\mu_{f_{R}}$ is the mean of $\mathcal{V} \sim f_{R}(v)$. It is interesting to note that $\mu_{\chi}(t)$ only depends on first-order moments of the other distributions. No other additional information on $g_{0}(x), f_{0}(v)$, and $f_{R}(v)$ is needed (such as higher-order moments) to establish the complete time evolution of $\mu_{\chi}(t)$. In passing, we note that making $\gamma=0$ in the first line of Eq. (10) leads to the expected result for a particle moving always at constant velocity, even though $\gamma$ is nonzero by definition (see Sec. II).

If $\mu_{f_{0}}=\mu_{f_{R}}=0$, then Eq. (10) implies constant $\mu_{\chi}(t)=$ $\mu_{g_{0}}$ for all $t \geqslant 0$. If $\mu_{f_{R}} \neq 0$, then the time evolution of the mean is more interesting. Figure 2 shows $\mu_{\chi}(t)$ for several choices of $\mu_{f_{0}} / \mu_{f_{R}}$. The initial behavior is determined by a competition between initial conditions and mean postcollision velocities. Then, when collisions have had enough time $\left(t \sim \gamma^{-1}\right)$ to randomize the motion, $\mu_{f_{R}}$ starts dominating. Finally, $\mu_{\chi}(t) \approx \mu_{f_{R}} t$ when $\gamma t \gg 1$ (irrespective of $\mu_{f_{0}}$ ).

We focus now on the variance $\sigma_{\chi}^{2}(t)$. For simplicity, we consider the case $\mu_{f 0}=\mu_{f_{R}}=0$. The general situation for nonzero values is discussed in Appendix C. Following a similar procedure as for the mean, we obtain

$$
\begin{aligned}
\sigma_{\chi}^{2}(t)= & \sigma_{g_{0}}^{2}+\frac{2 \sigma_{f_{0}}^{2}}{\gamma^{2}}\left[1-(1+\gamma t) e^{-\gamma t}\right] \\
& +\frac{2 \sigma_{f_{R}}^{2}}{\gamma^{2}}\left[-2+\gamma t+(2+\gamma t) e^{-\gamma t}\right] .
\end{aligned}
$$

This expression only depends on second-order moments of $g_{0}$, $f_{0}$, and $f_{R}$. Therefore, PDFs of very different form but similar mean and variance should lead to the same time evolution 

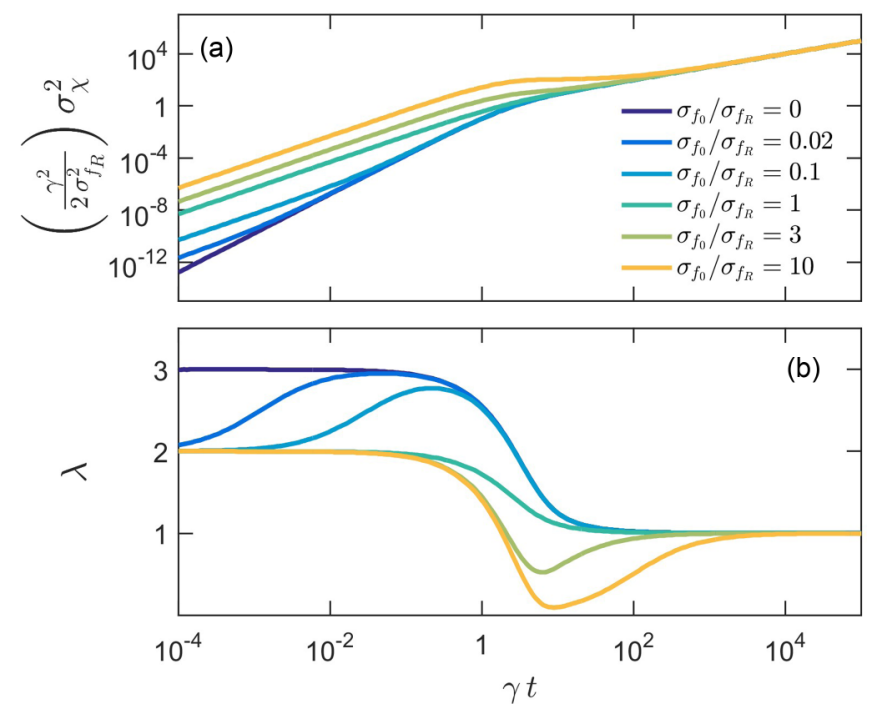

FIG. 3. (a) Evolution of $\sigma_{\chi}^{2}(t)$ for $\sigma_{g_{0}}=0, \mu_{f_{0}}=\mu_{f_{R}}=0$ and different values of $\sigma_{f_{0}} / \sigma_{f_{R}}$ as indicated in the legend. (b) Transport exponent $\lambda$ for the same cases (same color legend).

of $\sigma_{\chi}^{2}$. This is indeed verified using numerical simulations in Sec. III B. The case $\sigma_{f_{R}}=0$ corresponds to a Dirac-delta [30] distribution modeling only one possible post-collision velocity (in this case $v=0$, as we have chosen $\mu_{f_{R}}=0$ ). Equation (11) shows that, in that situation, $\sigma_{\chi}^{2}(t)$ tends to a constant when $\gamma t \gg 1$. If we consider now nonzero $\sigma_{f_{R}}$, then we can rewrite Eq. (11) as

$$
\begin{aligned}
\left(\frac{\gamma^{2}}{2 \sigma_{f_{R}}^{2}}\right) \sigma_{\chi}^{2}(t)= & \left(\frac{\gamma^{2}}{2 \sigma_{f_{R}}^{2}}\right) \sigma_{g_{0}}^{2}+\left(\frac{\sigma_{f_{0}}^{2}}{\sigma_{f_{R}}^{2}}-2+\gamma t\right) \\
& +e^{-\gamma t}\left[2-\frac{\sigma_{f_{0}}^{2}}{\sigma_{f_{R}}^{2}}+\gamma t\left(1-\frac{\sigma_{f_{0}}^{2}}{\sigma_{f_{R}}^{2}}\right)\right] .
\end{aligned}
$$

The RHS of this expression only depends on three parameters. The first one is $\gamma \sigma_{g_{0}} / \sigma_{f_{R}}$, a normalized initial width. The second one is $\gamma t$, the average number of collisions by time $t$. The third one is $\sigma_{f_{0}} / \sigma_{f_{R}}$, the ratio of the standard deviations of $\mathcal{V}_{0}$ and $\mathcal{V}$. The individual values of $\sigma_{f_{0}}$ and $\sigma_{f_{R}}$ do not play a role in the functional form of the time evolution of $\sigma_{\chi}^{2}$ (it is only their ratio that matters). We note that making $\sigma_{f_{0}} / \sigma_{f_{R}}=1$ in Eq. (12) leads to a result with the same functional form as the known solution for arbitrary times using the Langevin approach $[5,11]$.

Figure 3(a) shows the evolution of $\sigma_{\chi}^{2}$ when $\sigma_{g_{0}}=0$. This is the case when the initial location is known with complete certainty $\left[g_{0}(x)\right.$ is a Dirac-delta]. The behavior at long timescales (i.e., $\gamma t \gg 1$ ) is similar for all different values of $\sigma_{f_{0}} / \sigma_{f_{R}}$. It is in fact diffusive, as $\sigma_{\chi}^{2}$ is seen to scale linearly with time, i.e., $\sigma_{\chi}^{2} \sim t^{\lambda}$ with $\lambda=1$. We know this from computing the slope of the curves and verifying that in all cases it is equivalent to 1 for $\gamma t \gg 1$. In the log-log plot, the slope is in fact equivalent to $\lambda$. The number $\lambda$, which we call [3] transport exponent (or transport scaling [2]), may, however, take on other values

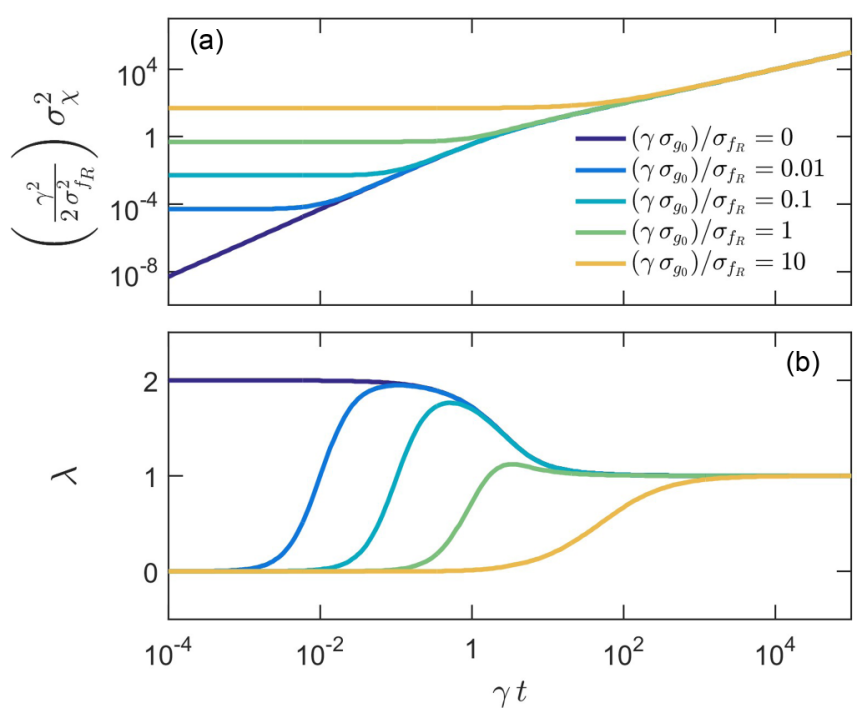

FIG. 4. (a) Evolution of $\sigma_{\chi}^{2}(t)$ for $\mu_{f_{0}}=\mu_{f_{R}}=0$ and different values of $\sigma_{g_{0}}$ as indicated in the legend. We use $\sigma_{f_{0}}=\sigma_{f_{R}}$ in all cases. (b) Transport exponent $\lambda$ of the same curves. Changes in normalized initial width $\left(\gamma \sigma_{g_{0}}\right) / \sigma_{f_{R}}$ have a significant effect on the slope at early times and could lead to misinterpretations of transport features if not properly considered. The case $\sigma_{g_{0}}=0$ is similar to the $\sigma_{f_{0}} / \sigma_{f_{R}}=1$ curve in Fig. 3.

when $\gamma t \lesssim 1$. Figure 3(a) shows that transport at early times can indeed be different from diffusive.

Figure 3(b) shows the time evolution of $\lambda$ for the same values of $\sigma_{f_{0}} / \sigma_{f_{R}}$ as in Fig. 3(a). We use

$$
\lambda(t)=\left[\frac{t}{\sigma_{\chi}^{2}(t)}\right] \frac{d}{d t}\left[\sigma_{\chi}^{2}(t)\right]
$$

to compute $\lambda$ from Eq. (12) (valid for $t>0$ ) and then plot it. We note that this definition of $\lambda$ uses the variance (some references may use a different convention $[2,3]$ ). There are several interesting observations. First, if $\sigma_{f_{0}} / \sigma_{f_{R}} \geqslant 1$, then the initial behavior is ballistic; i.e., $\lambda=2$. However, transport is significantly reduced at $\gamma t \approx 1$ and can become subdiffusive. In fact, we see that $\lambda$ can approach the value zero when $\sigma_{f_{0}} / \sigma_{f_{R}}>10$. The case $\sigma_{f_{0}}=\sigma_{f_{R}}$ models a situation similar [14] to PRW and indeed shows the same smooth transition from ballistic to diffusive around $\gamma t=1$. Probably more interesting is the case $\sigma_{f_{0}} / \sigma_{f_{R}}<1$ when $\gamma t \leqslant 1$. In that situation, $\lambda$ can attain values larger than 2 including $\lambda=3$. This superballistic transport, already observed in Ref. [19], is seen in Sec. IV A to be characteristic of velocity diffusion.

Figure 4 shows the evolution of $\sigma_{\chi}^{2}$ for fixed $\sigma_{f_{0}} / \sigma_{f_{R}}=$ 1 and different values of $\sigma_{g_{0}}$. Also shown is $\lambda$ which, for large normalized initial widths $\gamma \sigma_{g_{0}} / \sigma_{f_{R}}>1$, can completely change character from initially ballistic to no transport $(\lambda=$ $0)$. This exemplifies the importance of correctly accounting for $\sigma_{g_{0}}$ in transport studies at early times. In practice, it is not possible to know $\sigma_{g_{0}}$ exactly, so finite-width uncertainties are foreseen to always be present in practical applications which will mostly impact observations when $t<\gamma^{-1}$. 
The methods presented can be used to compute higherorder moments of $p(x, t)$ and find symbolic expressions for, for example, the skewness and kurtosis [28] of $\chi(t)$. As we are concerned with determining transport features, which involve the first and second moments, we do not undertake higherorder moment calculations here.

\section{B. Comparison of theory and numerical simulations}

We developed a simple code in MATLAB [31] (see Appendix D) that creates numerical estimates $m_{\chi}(t), s_{\chi}^{2}(t)$ of $\mu_{\chi}(t), \sigma_{\chi}^{2}(t)$, respectively.

We consider three possible choices of PDFs,

$$
\begin{aligned}
& \operatorname{Gaussian}\left(x ; \mu, \sigma^{2}\right)=\frac{1}{\sqrt{2 \pi \sigma^{2}}} e^{-\frac{(x-\mu)^{2}}{2 \sigma^{2}}}, \\
& \operatorname{Discrete}\left(x ; \mu, \sigma^{2}\right)=\frac{1}{2}[\delta(x-\mu-\sigma)+\delta(x-\mu+\sigma)], \\
& \operatorname{Uniform}\left(x ; \mu, \sigma^{2}\right)= \begin{cases}(2 \sqrt{3} \sigma)^{-1} & \text { if } \mu-\sqrt{3} \sigma \leqslant x \leqslant \mu+\sqrt{3} \sigma, \\
0 \text { otherwise, }\end{cases}
\end{aligned}
$$

for the initial conditions ( $g_{0}$ and $f_{0}$ ) and post-collision velocity distribution $\left(f_{R}\right)$.

In a given simulation, we choose one of three possible sets of values of $\gamma$ and $\sigma_{f_{R}}$, and one choice of PDFs. This means that we take $g_{0}, f_{0}$ and $f_{R}$ to be all Gaussian, all Uniform, or all Discrete. The particular selection is indicated with different markers in Fig. 5. Circles correspond to $\gamma=3 \mathrm{~s}^{-1}, \sigma_{f_{R}}=2 \mathrm{~m} / \mathrm{s}$ and Discrete PDFs. Triangles are $\gamma=1 \mathrm{~s}^{-1}, \sigma_{f_{R}}=1 \mathrm{~m} / \mathrm{s}$ and Gaussian PDFs. Crosses are $\gamma=$ $2 \mathrm{~s}^{-1}, \sigma_{f_{R}}=3 \mathrm{~m} / \mathrm{s}$ and Uniform distributions. We use in all cases $\mu_{f_{0}}=\mu_{f_{R}}=0$ and set $\sigma_{g_{0}}$ such that $\left(\gamma \sigma_{g_{0}}\right) / \sigma_{f_{R}}=10^{-4}$. The choice of a nonzero initial width is made to avoid otherwise large rounding errors in the variance, and consequently the transport exponent, if $\sigma_{g_{0}}$ is too close to zero near $t=0$.

The simulation results (see Fig. 5) show good agreement with the theory of Sec. III A. The evolution, in units of $\gamma t$, of the normalized variance [Fig. 5(b)] and the transport exponent [Fig. 5(c)] only depends on the ratio $\sigma_{f_{0}} / \sigma_{f_{R}}$ and is independent of the particular choices of $\gamma, \sigma_{f_{R}}$, and PDF shape, as expected. The increased variability of the markers in Fig. 5(a) when $\gamma t \gtrsim 10$ is due to the statistical error expected for the computation of the mean $m_{\chi}(t)$. This is illustrated in the case $\sigma_{f_{0}} / \sigma_{f_{R}}=1$ for which the markers lie within or near the shaded area representing the $1 \sigma$ region around $\mu_{\chi}(t)=0$.

\section{ADDITIVE VELOCITY JUMPS}

\section{A. Model}

We now consider the case

$$
f(u, v)=f_{A}(v-u),
$$

where $f_{A}$ is a PDF with finite first and second-order moments and can be Fourier-transformed. Upon a collision, the walker transitions to a new random velocity $u+\mathcal{V}$ where $\mathcal{V}$ is distributed $f_{A}$. Note that the new velocity depends on the velocity prior to the collision, and the dependency is additive. This choice models a generalized diffusion of velocities, as discussed in Appendix E, with a priori no limit on the maximum attainable speed. Relativistic effects are not included in the model, so nothing precludes reaching artificial supraluminal regimes after long enough times.

The analysis requires a different approach from the one used in Sec. III A. We take Eq. (5) and perform a Fourier transform with respect to the velocity variable $v$, using a convention similar to Eq. (3). We call $\beta$ the variable conjugate

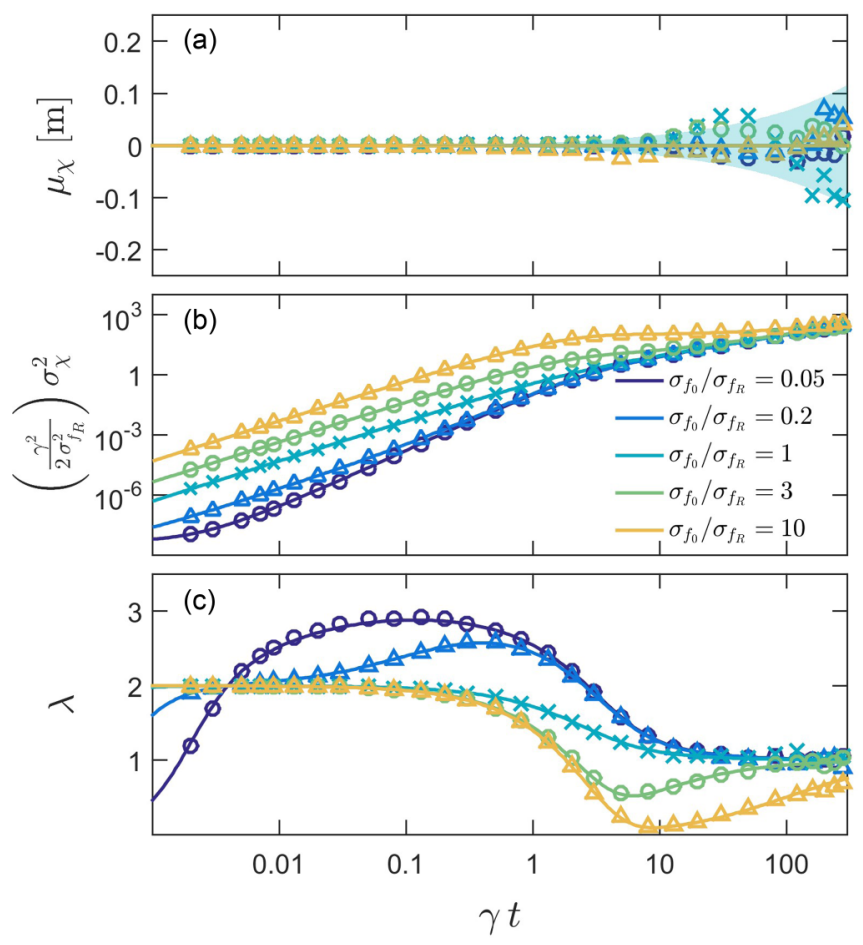

FIG. 5. Using $\left(\gamma \sigma_{g_{0}}\right) / \sigma_{f_{R}}=10^{-4}, \mu_{f_{0}}=\mu_{f_{R}}=0$ we compute (a) the mean $m_{\chi}(t)$, (b) the variance $s_{\chi}^{2}(t)$ and (c) the transport exponent of $\chi(t)$ at some sample times (markers) during simulations performed with different values of $\sigma_{f_{0}} / \sigma_{f_{R}}$ [colored according to the label in (b)]. Different markers (circles, triangles, crosses) are used to indicate one of three sets of parameter values of $\gamma, \sigma_{f_{R}}$ and choice of PDFs used in a particular simulation, as explained in the text. The solid lines are the theoretical values $\mu_{\chi}(t), \sigma_{\chi}^{2}(t)$ and $\lambda(t)$ from Sec. III A corresponding to the different $\sigma_{f_{0}} / \sigma_{f_{R}}$ (same color coding). There is good agreement between simulations and theory. 
to $v$. Then,

$$
\begin{aligned}
(s & \left.-\kappa \frac{\partial}{\partial \beta}+\gamma\right) p(\kappa, \beta, s)-g_{0}(\kappa) f_{0}(\beta) \\
& =\mathcal{F}\left\{\gamma \int f_{A}(v-u) p(\kappa, u, s) d u\right\} \\
& =\gamma f_{A}(\beta) p(\kappa, \beta, s) .
\end{aligned}
$$

As before, we use the transform variables ( $\kappa, \beta$ and/or $s$ ) to distinguish the transformed functions from the original ones. The last line in Eq. (16) follows from the known result for the transform of a convolution [30].

Evaluating Eq. (16) at $\kappa=0$, and solving for $\left.p(\kappa, \beta, s)\right|_{\kappa=0}$ we have

$$
\left.p(\kappa, \beta, s)\right|_{\kappa=0}=\frac{f_{0}(\beta)}{s+\gamma\left[1-f_{A}(\beta)\right]} .
$$

We can also rearrange and differentiate Eq. (16) with respect to $\kappa$, and then evaluate at $\kappa=0$. Then,

$$
\begin{aligned}
\{s & \left.+\gamma\left[1-f_{A}(\beta)\right]\right\}\left.\frac{\partial}{\partial \kappa} p(\kappa, \beta, s)\right|_{\kappa=0} \\
& =\left.f_{0}(\beta) \frac{\partial}{\partial \kappa} g_{0}(\kappa)\right|_{\kappa=0}+\left.\left[\frac{\partial}{\partial \beta} p(\kappa, \beta, s)\right]\right|_{\kappa=0} \\
& =-l \mu_{g_{0}} f_{0}(\beta)+\frac{\partial}{\partial \beta}\left[\left.p(\kappa, \beta, s)\right|_{\kappa=0}\right] .
\end{aligned}
$$

The first term in the last line follows from the relationship between the Fourier transform and the characteristic function of a PDF, as discussed in Eq. (7). The second term comes from the observation that, to compute a partial derivative, one treats other variables as constants, so the order of the derivative and the evaluation $\kappa=0$ can be interchanged. Replacing the term in parenthesis with the expression found in Eq. (17) and solving for $\left.\frac{\partial}{\partial \kappa} p(\kappa, \beta, s)\right|_{\kappa=0}$ we have

$$
\begin{aligned}
\left.\frac{\partial}{\partial \kappa} p(\kappa, \beta, s)\right|_{\kappa=0}= & \frac{-l \mu_{g_{0}} f_{0}(\beta)}{s+\gamma\left[1-f_{A}(\beta)\right]}+\frac{1}{s+\gamma\left[1-f_{A}(\beta)\right]} \\
& \times \frac{\partial}{\partial \beta}\left\{\frac{f_{0}(\beta)}{s+\gamma\left[1-f_{A}(\beta)\right]}\right\} .
\end{aligned}
$$

This expression can straightforwardly be used to compute $\mu_{\chi}(t)$ upon evaluating at $\beta=0$, in a similar fashion as in Eq. (6). Noticing that $\left.f_{A}(\beta)\right|_{\beta=0}=1$ and $\left.f_{0}(\beta)\right|_{\beta=0}=1$, we obtain

$$
\begin{aligned}
\mu_{\chi}(t) & =\mathcal{L}^{-1}\left\{\left.l \frac{\partial}{\partial \kappa} p(\kappa, \beta, s)\right|_{\substack{\kappa=0 \\
\beta=0}}\right\} \\
& =\mu_{g_{0}}+\mu_{f_{0}} t+\frac{\mu_{f_{A}}}{2} \gamma t^{2} .
\end{aligned}
$$

Figure 6 shows $\mu_{\chi}(t)$ for several choices of $\mu_{f_{0}} / \mu_{f_{A}}$. Although at $t \lesssim \gamma^{-1}$ the results are similar to those found in Sec. III A, later times show a clear difference wherein the mean tends to increase quadratically, irrespective of $\mu_{f_{0}}$, instead of linearly.

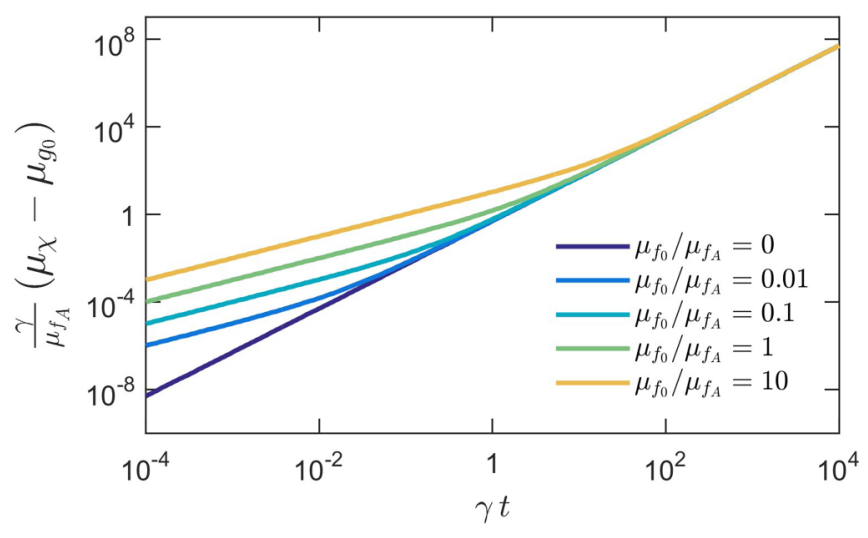

FIG. 6. Evolution of $\mu_{\chi}(t)$ in the case of additive velocity changes for different ratios $\mu_{f_{0}} / \mu_{f_{A}}$ assuming $\mu_{f_{A}}>0$. The functional form of $\left(\mu_{\chi}(t)-\mu_{g_{0}}\right)$ only depends on $\mu_{f_{0}} / \mu_{f_{A}}$ and $\gamma t$, as Eq. (20) shows.

To compute the variance, we differentiate Eq. (16) a second time with respect to $\kappa$ and evaluate at $\kappa=0$ to obtain

$$
\begin{aligned}
\{s & \left.+\gamma\left[1-f_{A}(\beta)\right]\right\}\left.\frac{\partial^{2}}{\partial \kappa^{2}} p(\kappa, \beta, s)\right|_{\kappa=0} \\
& =\left.f_{0}(\beta) \frac{\partial^{2}}{\partial \kappa^{2}} g_{0}(\kappa)\right|_{\kappa=0}+\left.2 \frac{\partial}{\partial \kappa}\left[\frac{\partial}{\partial \beta} p(\kappa, \beta, s)\right]\right|_{\kappa=0} \\
& =-\left(\sigma_{g_{0}}^{2}+\mu_{g_{0}}^{2}\right) f_{0}(\beta)+2 \frac{\partial}{\partial \beta}\left[\left.\frac{\partial}{\partial \kappa} p(\kappa, \beta, s)\right|_{\kappa=0}\right] .
\end{aligned}
$$

The last line in the expression above follows from considerations similar to the ones explained in the case of the mean [Eq. (20)], plus the assumption of continuity of the second derivatives to be able to interchange the order. Then, Eq. (19) can be inserted in the term in parenthesis, Eq. (21) can be evaluated at $\beta=0$, and $\left.\frac{\partial^{2}}{\partial \kappa^{2}} p(\kappa, \beta, s)\right|_{\kappa=0, \beta=0}$ can be solved for to obtain

$$
\begin{aligned}
\sigma_{\chi}^{2}(t) & =\mathcal{L}^{-1}\left\{-\left.\frac{\partial^{2}}{\partial \kappa^{2}} p(\kappa, \beta, s)\right|_{\substack{\kappa=0 \\
\beta=0}}\right\}-\left[\mu_{\chi}(t)\right]^{2} \\
& =\sigma_{g_{0}}^{2}+\sigma_{f_{0}}^{2} t^{2}+\frac{1}{3}\left(\sigma_{f_{A}}^{2}+\mu_{f_{A}}^{2}\right) \gamma t^{3} .
\end{aligned}
$$

Assuming now $\mu_{f_{A}}=0$ and $\sigma_{f_{A}}>0$, we can rewrite Eq. (22) as

$$
\left(\frac{\gamma^{2}}{2 \sigma_{f_{A}}^{2}}\right) \sigma_{\chi}^{2}(t)=\left(\frac{\gamma^{2}}{2 \sigma_{f_{A}}^{2}}\right) \sigma_{g_{0}}^{2}+\frac{1}{2}\left(\frac{\sigma_{f_{0}}^{2}}{\sigma_{f_{A}}^{2}}\right)(\gamma t)^{2}+\frac{1}{6}(\gamma t)^{3},
$$

which shows that at late times the variance scales as $t^{3}$, very different from the spatially diffusive behavior observed in Sec. III A when $t \gg \gamma^{-1}$. Transport is ballistic at early times, when the second term in the RHS dominates. These results can be seen in Fig. 7, where the time evolution of $\sigma_{\chi}^{2}$ is shown for different ratios $\sigma_{f_{0}} / \sigma_{f_{A}}$. Similarly as in Fig. 4, it can also happen that a finite initial width dominates the behavior at early times. If that is the case, then the transport exponent $\lambda$ can be brought all the way down to zero and a transition would slowly happen towards $\lambda=3$ to finally stay there. 

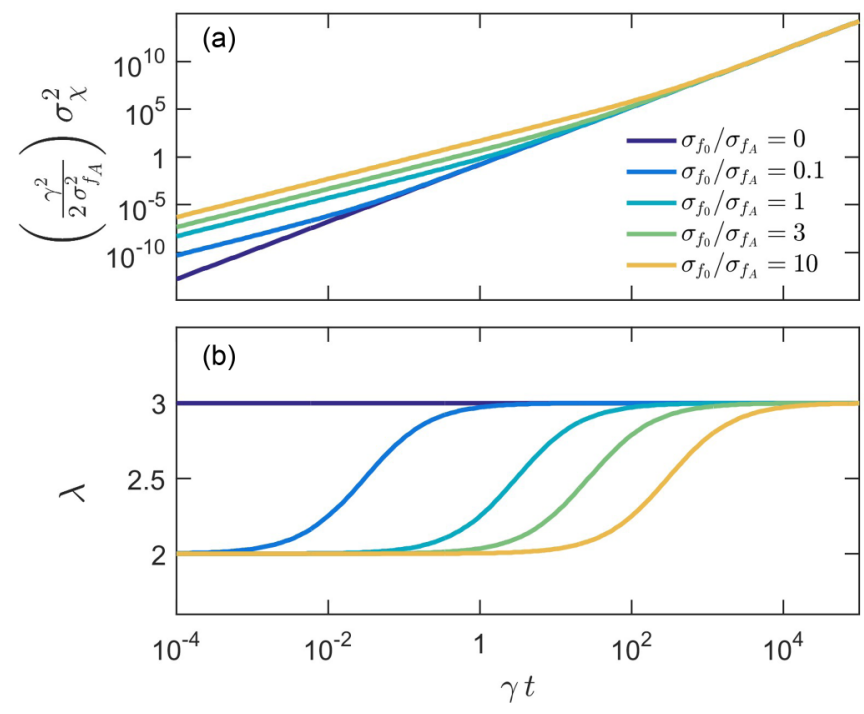

FIG. 7. (a) Evolution of $\sigma_{\chi}^{2}(t)$ for $\sigma_{g_{0}}=0, \mu_{f_{A}}=0$ and different values of $\sigma_{f_{0}} / \sigma_{f_{A}}$ as indicated in the legend. (b) Transport exponent $\lambda$ for the same cases (same color legend).

Comparing Fig. 7 to Fig. 3 in the cases $\sigma_{f_{0}} \ll \sigma_{f_{A}}$, one can find close similarities that suggest that the observation of $\lambda=$ 3 in Sec. III A may be related to diffusion of velocities at early times of the walk. This makes sense, as $u \approx 0$ at early times which makes $f_{A}(v-u) \approx f_{A}(v)$. Further dedicated studies are nevertheless required to validate this observation.

\section{B. Comparison with numerical simulations}

Simulations similar to those presented in Appendix D are performed to test the validity of the predictions of the additive velocity jump model. Upon a collision, the velocity of trajectory $n$ takes now the new value $v_{n}(t+\Delta t)=v_{n}(t)+\mathcal{V}_{n}$ with $\mathcal{V}_{n}$ distributed $f_{A}$. The time evolution algorithm [Eq. (D1)] is accordingly modified to

$$
\begin{aligned}
x_{n}(t+\Delta t) & =x_{n}(t)+v_{n}(t) \Delta t, \\
v_{n}(t+\Delta t) & = \begin{cases}v_{n}(t) & \text { if no collision happens, } \\
v_{n}(t)+\mathcal{V}_{n} & \text { if there is a collision. }\end{cases}
\end{aligned}
$$

Figure 8 shows the results of simulations performed with different choices of $\sigma_{f_{0}} / \sigma_{f_{A}}$. As before, we choose for each particular simulation one of three possible sets of values of parameters and distributions as indicated with crosses $(\gamma=$ $3 \mathrm{~s}^{-1}, \sigma_{f_{A}}=2 \mathrm{~m} / \mathrm{s}$, and Uniform PDFs), circles $\left(\gamma=1 \mathrm{~s}^{-1}\right.$, $\sigma_{f_{A}}=1 \mathrm{~m} / \mathrm{s}$, and Discrete PDFs) and triangles $\left(\gamma=2 \mathrm{~s}^{-1}\right.$, $\sigma_{f_{A}}=3 \mathrm{~m} / \mathrm{s}$, and Gaussian PDFs). Comparison with predictions from Sec. IV A (solid lines in Fig. 8) shows good agreement. The evolution, in units of $\gamma t$, of the normalized variance [Fig. 8(b)] and the transport exponent [Fig. 8(c)] only depends on the ratio $\sigma_{f_{0}} / \sigma_{f_{A}}$ and not on the particular choices of $\gamma, \sigma_{f_{A}}$, and PDF shape. The increased variability of $m_{\chi}(t)$ at late times in Fig. 8(a) is expected from statistical errors, as illustrated with the shaded area for the case $\sigma_{f_{0}} / \sigma_{f_{A}}=1$.

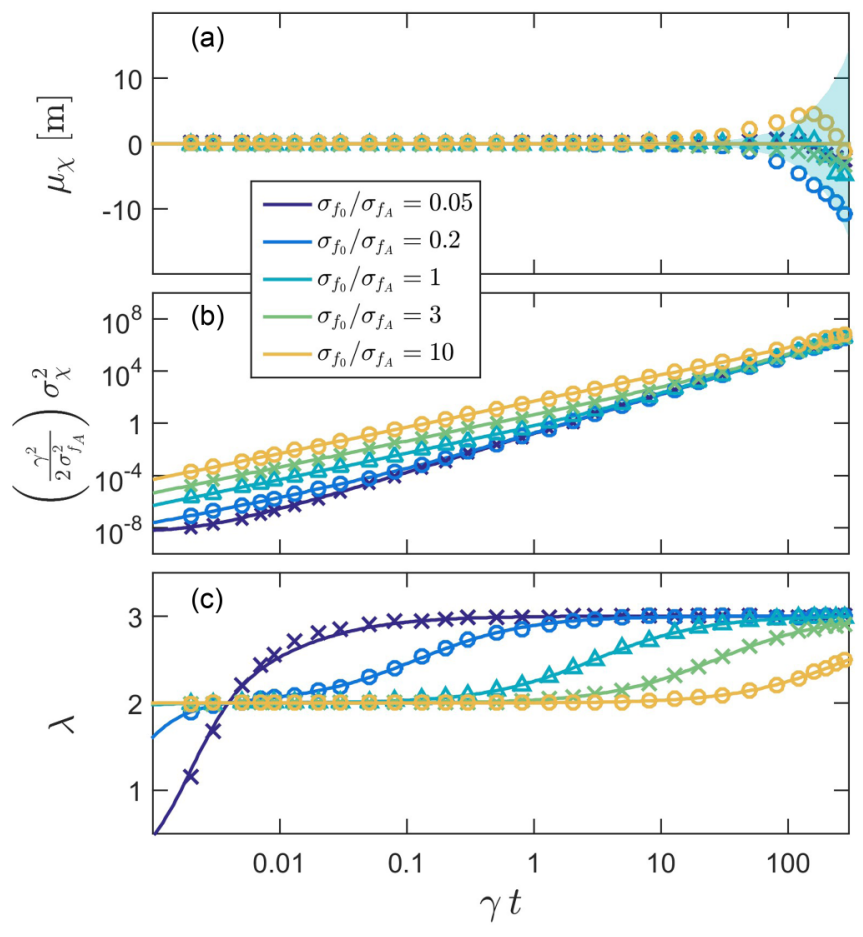

FIG. 8. Simulated (a) mean $m_{\chi}(t)$, (b) variance $s_{\chi}^{2}(t)$, and (c) transport exponent $\lambda(t)$ at some sample times (markers) during simulations performed with different values of $\sigma_{f_{0}} / \sigma_{f_{A}}$. As before, we use $\left(\gamma \sigma_{g_{0}}\right) / \sigma_{f_{A}}=10^{-4}$ (evident from the low $\lambda$ values at early times for $\left.\sigma_{f_{0}} / \sigma_{f_{A}}=0.05\right), \mu_{f_{0}}=\mu_{f_{A}}=0$ and different sets of parameters (different markers; see text). The solid lines are the theoretical values $\mu_{\chi}(t), \sigma_{\chi}^{2}(t)$, and $\lambda(t)$ from Sec. IV A (same color coding). There is good agreement between simulations and theory.

\section{SCALED VELOCITY CHANGES}

\section{A. Model}

We now study the situation where, upon a collision, the walker velocity becomes $v=c u$, a scaled version of the precollision velocity $u$. In this case, the velocity is multiplied by a constant factor that we call " $c$ " and require that it be nonzero (it can nevertheless be positive or negative). If $|c|>$ 1 , then the speed of the walker increases; if $|c|<1$, then the speed decreases; if $|c|=1$, then it remains unchanged. We have

$$
f(u, v)=\frac{1}{|c|} \delta\left(\frac{v}{c}-u\right),
$$

where the factor $1 /|c|$ is needed to fulfill the normalization condition $\int f(u, v) d v=1$ for all $u$ (see Sec. II). As with the case of additive velocities (Sec. IV A), we note that this model allows reaching supraluminal speeds when $|c|>1$, a feature that poses limitations on its applicability.

With this choice of $f$, Eq. (2) becomes

$$
\left(\frac{\partial}{\partial t}+v \frac{\partial}{\partial x}+\gamma\right) p(x, v, t)=\frac{\gamma}{|c|} p\left(x, \frac{v}{c}, t\right) .
$$

This is an interesting equation where the second component of $p$ appears scaled on the RHS compared to the LHS. Following an approach similar to the one used in Sec. IV A to determine the statistics of $\chi(t)$, we Fourier transform this expression in 
$x$ and $v$ and Laplace transform in $t$ to obtain

$$
\left(s-\kappa \frac{\partial}{\partial \beta}+\gamma\right) p(\kappa, \beta, s)-g_{0}(\kappa) f_{0}(\beta)=\gamma p(\kappa, c \beta, s) .
$$

Evaluation of this equation at $\kappa=0$ leads to

$$
\left.(s+\gamma) p(\kappa, \beta, s)\right|_{\kappa=0}-f_{0}(\beta)=\left.\gamma p(\kappa, c \beta, s)\right|_{\kappa=0},
$$

which can be differentiated with respect to $\beta$ and evaluated at $\beta=0$ to yield

$$
\left.\frac{\partial}{\partial \beta} p(\kappa, \beta, s)\right|_{\substack{\kappa=0 \\ \beta=0}}=\frac{-\imath \mu_{f_{0}}}{s+(1-c) \gamma} .
$$

Equation (29) uses $\left.\frac{\partial f_{0}(\beta)}{\partial \beta}\right|_{\beta=0}=-\imath \mu_{f_{0}}$ and the relationship

$$
\left.\frac{\partial}{\partial \beta}\left[\left.p(\kappa, c \beta, s)\right|_{\kappa=0}\right]\right|_{\beta=0}=\left.c \frac{\partial}{\partial \beta} p(\kappa, \beta, s)\right|_{\substack{\kappa=0 \\ \beta=0}}
$$

which comes from noticing that $\left.c \beta\right|_{\beta=0}=\left.\beta\right|_{\beta=0}=0$ for any bounded value of $c$.

Meanwhile, differentiating Eq. (27) with respect to $\kappa$ and evaluating at $\kappa=0$ leads to

$$
\begin{aligned}
(s & +\gamma)\left.\frac{\partial}{\partial \kappa} p(\kappa, \beta, s)\right|_{\kappa=0}-\left.\gamma \frac{\partial}{\partial \kappa} p(\kappa, c \beta, s)\right|_{\kappa=0} \\
& =-l \mu_{g_{0}} f_{0}(\beta)+\frac{\partial}{\partial \beta}\left[\left.p(\kappa, \beta, s)\right|_{\kappa=0}\right] .
\end{aligned}
$$

Evaluating this expression at $\beta=0$ and replacing Eq. (29) in the RHS of Eq. (30), we get

$$
\left.s \frac{\partial}{\partial \kappa} p(\kappa, \beta, s)\right|_{\substack{\kappa=0 \\ \beta=0}}=-l \mu_{g_{0}}-\frac{\imath \mu_{f_{0}}}{s+(1-c) \gamma},
$$

which leads to

$$
\begin{aligned}
& \mu_{\chi}(t)=\mathcal{L}^{-1}\left\{\left.l \frac{\partial}{\partial \kappa} p(\kappa, \beta, s)\right|_{\kappa=0}\right\}, \\
& =\mu_{g_{0}}+\mu_{f_{0}}\left[\frac{e^{(c-1) \gamma t}-1}{(c-1) \gamma}\right] \text {. }
\end{aligned}
$$

Equation (32) assumes $c \neq 1$. That case, however, is easily obtainable by evaluating $c$ in Eq. (31) before taking the inverse Laplace transform. Doing so leads to the expected result for collision-free dynamics.

Figure 9 shows $\mu_{\chi}(t)$ for several choices of $c$. We assume $\mu_{f_{0}} \neq 0$ (the zero case is trivial) and use $\mu_{f_{0}}$ for normalization. For $c>1$ the mean exhibits exponential growth. The case $c<$ 1 shows an initially linear growth that nevertheless stagnates. These observations are different from the ones obtained in Secs. III and IV and further show that different types of velocity transitions can lead to different time evolution features of the statistics of $\chi$.

To compute the variance we differentiate Eq. (27) a second time with respect to $\kappa$ and evaluate at $\kappa=0$ and $\beta=0$.

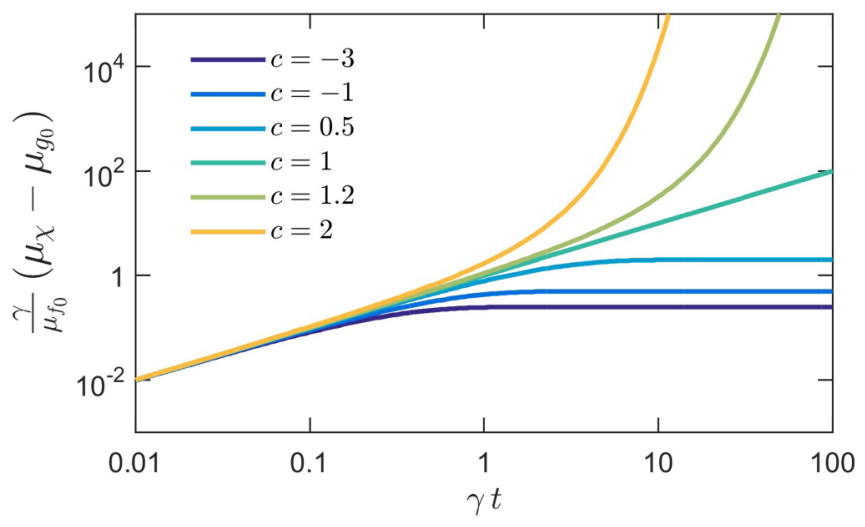

FIG. 9. Evolution of $\mu_{\chi}(t)$ in the case of scaled velocity changes for different values of $c$. The mean is normalized using $\mu_{f_{0}}$ which is different from previous sections.

Rearranging terms leads to

$$
\begin{aligned}
& \left.s \frac{\partial^{2}}{\partial \kappa^{2}} p(\kappa, \beta, s)\right|_{\substack{\kappa=0 \\
\beta=0}} \\
& \quad=-\left(\sigma_{g_{0}}^{2}+\mu_{g_{0}}^{2}\right)+\left.2 \frac{\partial}{\partial \beta}\left[\left.\frac{\partial}{\partial \kappa} p(\kappa, \beta, s)\right|_{\kappa=0}\right]\right|_{\beta=0} .
\end{aligned}
$$

The second term on the RHS can be computed in the following way. We differentiate Eq. (30) with respect to $\beta$ and evaluate at $\beta=0$. This yields

$$
\begin{aligned}
\left.(s+\gamma(1-c)) \frac{\partial}{\partial \beta}\left[\left.\frac{\partial}{\partial \kappa} p(\kappa, \beta, s)\right|_{\kappa=0}\right]\right|_{\beta=0} \\
=-\mu_{g_{0}} \mu_{f_{0}}+\left.\frac{\partial^{2}}{\partial \beta^{2}}\left[\left.p(\kappa, \beta, s)\right|_{\kappa=0}\right]\right|_{\beta=0} .
\end{aligned}
$$

The term involving the second derivative in $\beta$ can be computed by differentiating Eq. (28) twice and evaluating at $\beta=$ 0 . Collecting terms, we obtain

$$
\left.\frac{\partial^{2}}{\partial \beta^{2}}\left[\left.p(\kappa, \beta, s)\right|_{\kappa=0}\right]\right|_{\beta=0}=-\frac{\sigma_{f_{0}}^{2}+\mu_{f_{0}}^{2}}{s+\gamma\left(1-c^{2}\right)} .
$$

Replacing this expression in Eq. (34), we solve for the mixed derivative term and replace it in Eq. (33). We then obtain the variance the same way as in Eq. (22). For simplicity, we show the results for the case $\mu_{f_{0}}=\mu_{g_{0}}=0$, although the procedure above can be used to find the general situation with nonzero means of the initial conditions. We have

$$
\sigma_{\chi}^{2}(t)=\sigma_{g_{0}}^{2}+\frac{2 \sigma_{f_{0}}^{2}}{(c-1)^{2} \gamma^{2}}\left[\frac{1}{c+1}-\frac{e^{(c-1) \gamma t}}{c}+\frac{e^{\left(c^{2}-1\right) \gamma t}}{c^{2}+c}\right],
$$

where we have assumed $c \neq 1$ and $c \neq-1$. These two cases can be straightforwardly obtained by replacing the values of $c$ directly in Eqs. (33)-(35) and then solving for $\sigma_{\chi}^{2}(t)$. If $c=$ 1 , then one obtains $\sigma_{\chi}^{2}(t)=\sigma_{g_{0}}^{2}+\sigma_{f_{0}}^{2} t^{2}$, as expected for the collisionless case, since the walker does not change velocity with collisions. The case $c=-1$ corresponds to the situation where the walker reverses direction upon each collision. This is very similar to the 1D PRW model (see Sec. I) and, indeed, 

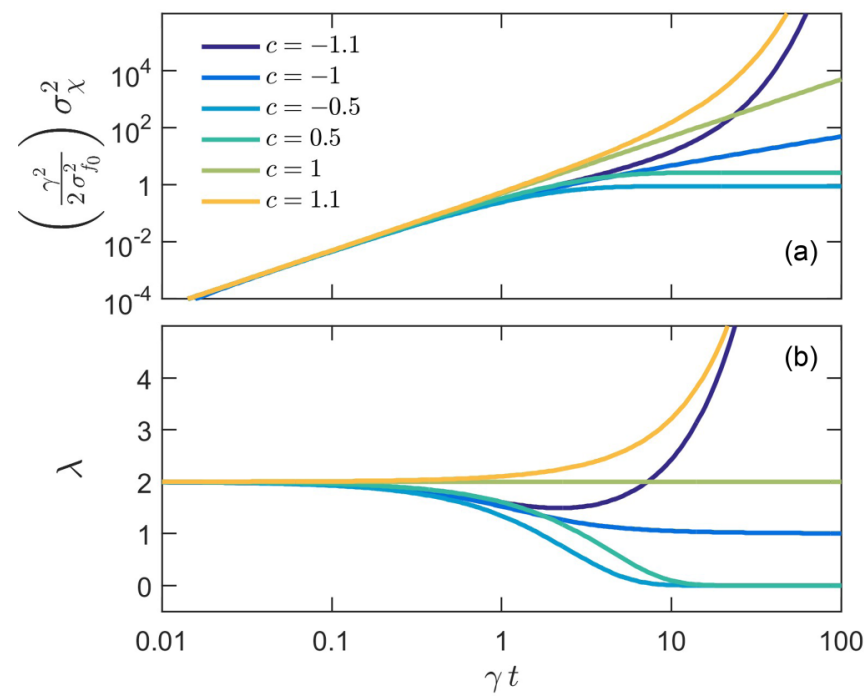

FIG. 10. (a) Evolution of $\sigma_{\chi}^{2}(t)$ in the case of scaled velocity changes for different values of $c$. We assume here $\sigma_{g_{0}}=0$ and $\mu_{f_{0}}=0$, although the procedure described in the text can allow us to find explicit results for nonzero cases. Notice that the variance is normalized using $\sigma_{f_{0}}$. (b) Transport exponents corresponding to the curves in (a).

one obtains the similar result

$$
\sigma_{\chi}^{2}(t)=\sigma_{g_{0}}^{2}+\frac{\sigma_{f_{0}}^{2}}{2 \gamma^{2}}\left(-1+2 \gamma t+e^{-2 \gamma t}\right) .
$$

This expression, though, takes into account the probabilistic nature of the initial conditions instead of using a constant speed parameter as in 1D PRW $[13,14,32]$. Note that we are assuming here $\mu_{g_{0}}=\mu_{f_{0}}=0$.

Figure 10 shows predictions of $\sigma_{\chi}^{2}$ as a function of time for several values of $c$. At early times, when few collisions are expected to have happened, all values of $c$ lead to ballistic $(\lambda=2)$ behavior. This ceases to be the case as one approaches $t=\gamma^{-1}$. The speed loss (or gain) caused by the collisions starts decelerating (accelerating) the walker and forces it to quickly come to a halt (exponentially increase) after only a few collisions $(\gamma t \approx 10)$. Speed losses of $50 \%$ and gains of only $10 \%$ per collision lead to the results shown in the figure. Equation (36) can nevertheless be used for arbitrary values of $c$ that can lead to faster drops (rises) in $\lambda$. There is also a visible difference when collisions cause changes in direction $(c<0)$. These differences, however, tend to be less pronounced at long timescales (except for the case $c=-1$ as described above).

Going back to Eq. (26), we can consider the general case of nonzero initial means to arrive at an interesting result for the case $c=-1$. Replacing this value of $c$ in the equation we get

$$
\begin{aligned}
& \left(\frac{\partial}{\partial t}+v \frac{\partial}{\partial x}+\gamma\right) p(x, v, t)=\gamma p(x,-v, t) \\
& \left(\frac{\partial}{\partial t}-v \frac{\partial}{\partial x}+\gamma\right) p(x,-v, t)=\gamma p(x, v, t),
\end{aligned}
$$

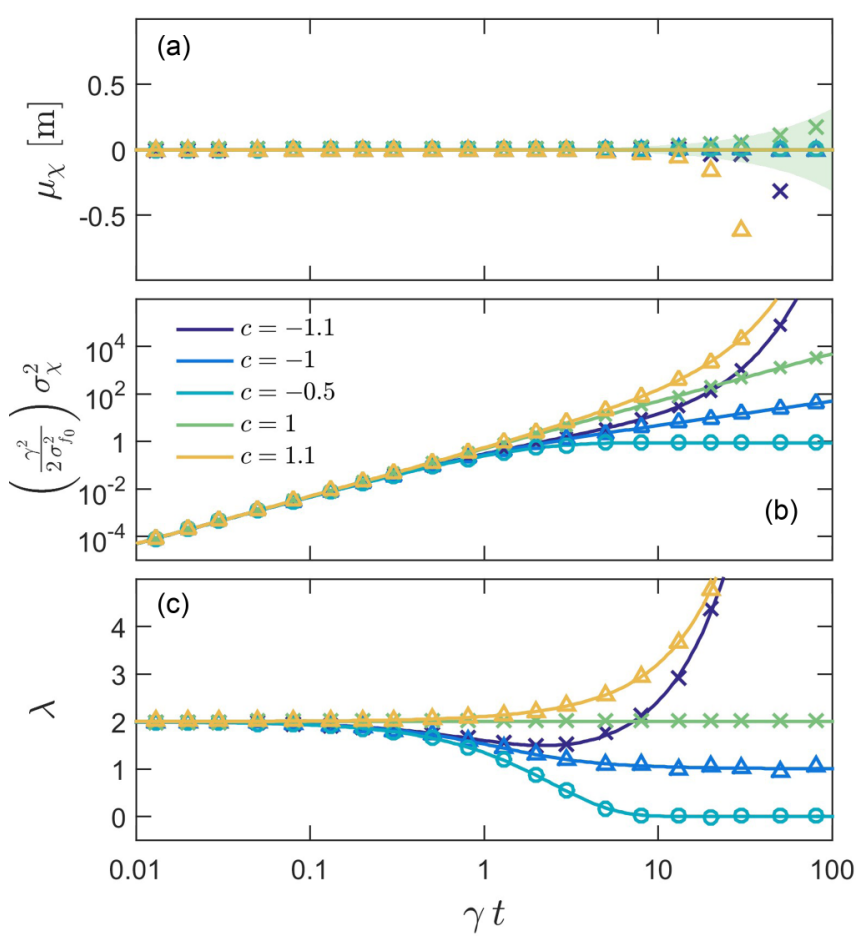

FIG. 11. Simulated (a) mean $m_{\chi}(t)$, (b) variance $s_{\chi}^{2}(t)$, and (c) transport exponent $\lambda(t)$ at some sample times (markers) during simulations performed with different values of $c$. We use $\left(\gamma \sigma_{g_{0}}\right) / \sigma_{f_{0}}=10^{-4}, \mu_{g_{0}}=0, \mu_{f_{0}}=0$ and different sets of parameters (different markers; see text). The solid lines are the theoretical values $\mu_{\chi}(t), \sigma_{\chi}^{2}(t)$, and $\lambda(t)$ from Sec. V A (same color coding). There is good agreement between simulations and theory.

where the second line is equivalent to the first one, only evaluated at $-v$. By combining the two expressions, we obtain the single equation

$$
\left(\frac{\partial^{2}}{\partial t^{2}}+2 \gamma \frac{\partial}{\partial t}\right) p(x, v, t)=v^{2} \frac{\partial^{2}}{\partial x^{2}} p(x, v, t),
$$

which is similar to the TE $[9,13,14]$ except that the velocity is an independent variable and not a fixed parameter.

\section{B. Comparison with numerical simulations}

We run simulations similar to those of Secs. III B and IV B. Here, though, the velocity of trajectory $n$ takes the new value $v_{n}(t+\Delta t)=c v_{n}(t)$ upon a collision. In contrast to Eq. (D1), the time evolution algorithm is

$$
\begin{aligned}
& x_{n}(t+\Delta t)=x_{n}(t)+v_{n}(t) \Delta t, \\
& v_{n}(t+\Delta t)= \begin{cases}v_{n}(t) & \text { if no collision happens, } \\
c v_{n}(t) & \text { if there is a collision. }\end{cases}
\end{aligned}
$$

Figure 11 shows the results of simulations performed for different values of $c$. As in previous sections, we choose for each particular simulation one of three possible sets of values of parameters and distributions as indicated with crosses $(\gamma=$ $1 \mathrm{~s}^{-1}, \sigma_{f_{0}}=1 \mathrm{~m} / \mathrm{s}$ and Uniform PDFs $)$, triangles $\left(\gamma=3 \mathrm{~s}^{-1}\right.$, $\sigma_{f_{0}}=2 \mathrm{~m} / \mathrm{s}$ and Gaussian PDFs $)$, and circles $\left(\gamma=2 \mathrm{~s}^{-1}\right.$, $\sigma_{f_{0}}=3 \mathrm{~m} / \mathrm{s}$ and Discrete PDFs). As seen in the figure, there is good agreement of the sample statistics with the predictions 
from Sec. V A (solid lines) of normalized variance [Fig. 11(b)] and transport exponent [Fig. 11(c)]. There is also good agreement of the means, as shown in Fig. 11(a). The deviations from zero mean at late times are expected from statistical errors, as illustrated by the shaded area in the case $c=1$.

When $|c| \gtrsim 1.5$ (not shown), the simulations produce results appreciably different from theoretical predictions at times $t>\gamma^{-1}$. The value of the simulated variance, for example, is consistently lower than that expected from Eq. (36). The difference becomes more pronounced as $|c|$ is made larger. The problem is likely due to contributions to the mean and variance of very rare events, whereby the walker undergoes many more collisions than the average. The walker can reach extremely large $x$ that yields finite results upon multiplication with the very low probabilities of occurrence. The low values of probability make the events impossible to resolve with the finite number of trajectories considered in this work $\left(10^{5}\right.$; see Appendix D). Separate studies suggest that the required sample size is many orders of magnitude larger (already for $|c|=2$ ), making the use of our simulations impractical in these situations. The studies show, however, that accounting for the rare events leads to the expected theoretical scaling given by Eq. (36).

\section{CONCLUSIONS}

Borrowing some ideas from PRW $[9,10,15]$ and biological dispersion [7], we have developed a technique to find exact analytic expressions for the mean, variance and transport exponent of a 1D random walker subject to Poisson-distributed collisions (with constant rate) which moves at constant velocity between collisions. Transport properties, as established with the value of the transport exponent $\lambda$ [Eq. (13)], have in general been shown to be time-varying and dependent on the possibly random initial conditions of location and velocity.

Three different types of velocity transitions are studied. In Sec. III we look at the case where the walker acquires a random velocity upon each collision and the new velocity is independent (in the statistical sense) from the previous state. The results show a diffusive behavior at long timescales $t \gg \tau$ (remembering that $\tau \equiv \gamma^{-1}$ ), but ballistic $(\lambda=2)$ and even superballistic $(\lambda>2)$ transport can be observed at early times $t \lesssim \tau$, depending on the initial velocity. Transport can also become subdiffusive $(\lambda<1)$ when $\tau \lesssim t \lesssim 100 \tau$ if the typical initial velocity is larger than the post-collision one. The initial walker location width also plays a role at early times. It is observed that $\lambda$ can come close to zero if the typical distance traveled between collisions is short compared to the standard deviation of the initial location.

One interesting observation is that, with the right choice of initial and post-collision velocity PDFs, the system can be made to always have bounded velocities. This is a concern in CTRW which is therefore avoided here. Furthermore, the predictions of $\lambda$ only depend on first and second moments of initial and post-collision velocity PDFs. This prevents requiring more detailed knowledge (i.e., higher-order moments) of the distributions to determine transport properties. Another interesting observation is that, in some cases, diffusive transport behavior is reached only after a significant time has elapsed.
For example, one must wait for $t \geqslant 100 \tau$ to reach $\lambda \approx 1$ whenever $\sigma_{f_{0}} / \sigma_{f} \geqslant 3$ (see Fig. 3).

In Sec. IV we study additive collisions where the postcollision velocity is the result of adding a random value to the precollision velocity. As argued in Appendix E, this situation models a generalized diffusion of velocities. Transport at early times shows similarities to the results in Sec. III when typical post-collision velocities are larger than initial ones. In those cases, ballistic and superballistic regimes are observed. Behavior for $t \gg \tau$ is very different from before though, with $\lambda \rightarrow 3$ for all values of initial velocity. The superballistic regime at long timescales is artificial in that it ultimately leads to unbounded velocities. Relativistic effects should be incorporated in the model if ever one is interested in obtaining physical predictions at very long $t$.

Last, we explore scaled velocity changes. In that case, the walker velocity is multiplied by a constant factor $c$ at each collision. This leads to very different results from before, with transport increasing exponentially when $|c|>1$ or becoming zero when $t \gg \tau$ and $|c|<1$. The case $c=-1$ is interesting as it establishes a connection to PRW, yielding results similar to what would be expected from that formalism.

Although we focus on first and second-order moments (relevant to transport studies), the procedures presented here can straightforwardly be extended to higher-order statistics of the random walker location (as a function of time), for example, the skewness and kurtosis. The technique can also be extended to higher dimensions for the three types of collisions presented. Higher dimensions, though, allow for more complex types of collisions that require dedicated analyses. These will be the subject of future studies.

\section{ACKNOWLEDGMENTS}

This work has been carried out within the framework of the EUROfusion Consortium and has received funding from the Euratom research and training programme 2014-2018 and 2019-2020 under Grant Agreement No. 633053. The views and opinions expressed herein do not necessarily reflect those of the European Commission. This work was supported in part by the Swiss National Science Foundation.

\section{APPENDIX A: TRANSPORT EQUATION}

To start, we borrow some basic ideas from PRW and biological dispersal. In particular, we follow some basic steps in the approach described in Refs. [7,9,10,15]. Many other methods have been developed to investigate similar situations in PRW [13,14,32,33] but are not pursued here.

Let $p_{v}(x, t)$ be the PDF of the random 1D location $\chi(t)$ of a walker moving with velocity $v$ along " $x$." Then, for given $x=X$ and $t=T, p_{v}(X, T) d x$ is the probability of finding the walker, which moves with velocity $v$, in the interval $(X, X+d x)$ at time $T$. We assume, for now, that the set $\mathrm{V}$ of possible velocities is discrete and allowed to have an arbitrary number of nonrepeated elements (possibly infinite). This is different from PRW, where $\mathrm{V}=\left\{v_{0},-v_{0}\right\}$ with $v_{0}>$ 0 a real parameter. In that case, the walker only has two possible motion states (towards increasing $x$ or decreasing $x$, respectively). 
The total probability of finding the walker in the interval $(X, X+d x)$ at time $T$ is the sum of probabilities corresponding to the mutually exclusive events of being in a different motion state (i.e., having a particular velocity) at the same location. Thus, $p(x, t)=\sum_{v} p_{v}(x, t)$ where the sum is performed over all possible velocities $v \in \mathrm{V}$.

The walker keeps the same $v$ unless it undergoes a collision. Collisions happen after exponentially distributed waiting times with mean $\tau$, or equivalently, the number of collisions is distributed Poisson $(t / \tau)$, so the probability that the walker undergo a collision in a short time interval $d t \rightarrow 0$ is $d t / \tau$. Indeed, obtaining $t / \tau$ collisions by time $t$ can be seen as successes in $t / d t$ Bernoulli trials [33] with probability of success $(t / \tau) /(t / d t)=d t / \tau$. The number of collisions is then distributed Binomial $(t / d t, d t / \tau)$ which, for short $d t$, converges to Poisson $(t / \tau)[28,33]$.

We now define the collision rate $\gamma=1 / \tau$. This is the typical number of collisions experienced by the walker per unit time. We assume $\gamma$ to be constant in time and independent of location and velocity. We also define the transition probabilities $f_{u \rightarrow v}$. These are the probabilities of going from a state of velocity " $u$ " to a state " $v$ " given that a collision has happened. They are parameters external to the model which must fulfill the normalization condition $\sum_{v} f_{u \rightarrow v}=1$ for all $u \in \mathrm{V}$, so that a walker existing in a state " $u$ " is not lost upon a collision. The probability of being in state " $v$ " at time $t+d t$ is then

$$
\begin{aligned}
p_{v}(x, t+d t)= & \overbrace{p_{v}(x-v d t, t)\left[1-\gamma d t\left(\sum_{u \neq v} f_{v \rightarrow u}\right)\right]}^{\text {Probability of staying in " } v "} \\
& +\overbrace{\sum_{u \neq v}\left[p_{u}(x-u d t, t) \gamma d t f_{u \rightarrow v}\right]}^{\text {Prob. of transitioning in from " } u \text { " }} \\
= & p_{v}(x-v d t, t)(1-\gamma d t) \\
& +\gamma d t \sum_{u}\left[p_{u}(x-u d t, t) f_{u \rightarrow v}\right]
\end{aligned}
$$

where in the last line $u$ runs over all possible velocities (including $v$ ). To simplify notation, we define the function $f: \mathrm{V} \times \mathrm{V} \rightarrow[0,1], f(u, v)=f_{u \rightarrow v}$ such that $\sum_{v} f(u, v)=$ 1 for all $u$.

Now $p_{v}(x, t+d t)=p_{v}(x, t)+\frac{\partial p_{v}(x, t)}{\partial t} d t+O\left(d t^{2}\right)$ and $p_{v}(x-v d t, t)=p_{v}(x, t)-\frac{\partial p_{v}(x, t)}{\partial x} v d t+O\left(d t^{2}\right)$. Replacing in Eq. (A1), neglecting terms of $O\left(d t^{2}\right)$ and rearranging leads to

$$
\left(\frac{\partial}{\partial t}+v \frac{\partial}{\partial x}+\gamma\right) p_{v}(x, t)=\gamma \sum_{u} f(u, v) p_{u}(x, t) .
$$

This expression is a set of equations that governs the time evolution of $p_{v}(x, t)$ for all $v$, with the number of equations being equivalent to $|\mathrm{V}|$. Notice that the $p_{v}(x, t)$ are coupled through the right-hand side (RHS), so it is in general difficult to find a solution whenever $|\mathrm{V}|$ is large (or infinite).
More generally, the possible velocities may form a continuum. We can then rewrite Eq. (A2) as

$$
\left(\frac{\partial}{\partial t}+v \frac{\partial}{\partial x}+\gamma\right) p(x, v, t)=\gamma \int f(u, v) p(x, u, t) d u \text {. }
$$

Here, however, the interpretation of some of the quantities must change. $f$ is now a function $f: \mathbb{R}^{2} \rightarrow[0, \infty)$ such that $f(u, v) d v$ is the probability that a walker with velocity $u$ transitions to a velocity in $(v, v+d v)$ upon a collision. It must satisfy the normalization condition $\int f(u, v) d v=1$ for all $u$ to conserve probability. The function $p$ with $x=X, t=T$ fixed must be regarded as a density, so that $p(X, V, T) d x d v$ is the probability of finding the walker with some velocity in $(V, V+d v)$ at the location $(X, X+d x)$ at time $T$.

Equation (2), which is the same as Eq. (A3), is a 1D transport equation [27] with collisions-distributed Poisson (with constant rate).

\section{APPENDIX B: DETERMINATION OF THE MEAN FOR THE CASE OF RANDOM VELOCITY UPON A COLLISION}

To compute the mean, as established with Eq. (7), we need to differentiate $p(\kappa, s)$ [given by Eq. (9)] with respect to $\kappa$ and then evaluate the result at $\kappa=0$.

Normalization of the PDFs implies $\int f_{R}(v) d v=1$, $\int f_{0}(v) d v=1$, and $\left.g_{0}(\kappa)\right|_{\kappa=0}=1$. Also, by the same argument leading to Eq. (7), we have $\left.\frac{\partial}{\partial \kappa} g_{0}(\kappa)\right|_{\kappa=0}=-\imath \mu_{g_{0}}$, where $\mu_{g_{0}}$ is the mean of $g_{0}(x)$. Then

$$
\left.\frac{\partial}{\partial \kappa} p(\kappa, s)\right|_{\kappa=0}=-\imath \frac{\mu_{g_{0}}}{s}-\imath \frac{s \int v f_{0}(v) d v+\gamma \int v f_{R}(v) d v}{s^{2}(s+\gamma)} .
$$

However, $\int v f_{0}(v) d v \equiv \mu_{f_{0}}$ and $\int v f_{R}(v) d v \equiv \mu_{f_{R}}$ by definition of the mean of the PDFs $f_{0}$ and $f_{R}$, respectively. Therefore,

$$
\left.\imath \frac{\partial}{\partial \kappa} p(\kappa, s)\right|_{\kappa=0}=\frac{\mu_{g_{0}}}{s}+\frac{s \mu_{f_{0}}+\gamma \mu_{f_{R}}}{s^{2}(s+\gamma)},
$$

which straightforwardly leads to Eq. (10).

\section{APPENDIX C: DETERMINATION OF THE VARIANCE FOR THE CASE OF RANDOM VELOCITY UPON A COLLISION}

Referring again to Eq. (7), the computation of the variance of $\chi$ requires evaluating the second derivative of $p(\kappa, s)$ with respect to $\kappa$ and then making $\kappa=0$. In addition to the normalization conditions stated in Appendix B, we notice that

$$
\begin{aligned}
& \int v^{2} f_{R}(v) d v=\sigma_{f_{R}}^{2}+\mu_{f_{R}}^{2}, \\
& \int v^{2} f_{0}(v) d v=\sigma_{f_{0}}^{2}+\mu_{f_{0}}^{2}, \\
& \left.\frac{\partial^{2}}{\partial \kappa^{2}} g_{0}(\kappa)\right|_{\kappa=0}=-\sigma_{g_{0}}^{2}-\mu_{g_{0}}^{2},
\end{aligned}
$$

where the first two lines come from the definition of the variance of $\mathcal{V}$ and $\mathcal{V}_{0}$ (with PDFs $f_{R}$ and $f_{0}$, respectively) and the last line comes from the relationship between the characteristic function of $g_{0}(x)$ and the second moment of $\chi_{0}$ [see discussion leading to Eq. (7)]. 
Starting from the expression for $p(\kappa, s)$ in Eq. (9), we perform the different calculations [34], replacing with the expressions in Eq. $(\mathrm{C} 1)$ when necessary, and then take the inverse Laplace transform. Finally, we use the result for $\mu_{\chi}(t)$ in Appendix B and simplify to obtain the following general expression for the variance of $\chi$,

$$
\begin{aligned}
\sigma_{\chi}^{2}(t)= & \mathcal{L}^{-1}\left\{-\left.\frac{\partial^{2}}{\partial \kappa^{2}} p(\kappa, s)\right|_{\kappa=0}\right\}-\left[\mu_{\chi}(t)\right]^{2} \\
= & \sigma_{g_{0}}^{2}+\frac{\left(\mu_{f_{R}}-\mu_{f_{0}}\right)^{2}}{\gamma^{2}}\left(1-2 \gamma t e^{-\gamma t}-e^{-2 \gamma t}\right) \\
& +\frac{2 \sigma_{f_{R}}^{2}}{\gamma^{2}}\left[-2+\gamma t+(2+\gamma t) e^{-\gamma t}\right] \\
& +\frac{2 \sigma_{f_{0}}^{2}}{\gamma^{2}}\left[1-(1+\gamma t) e^{-\gamma t}\right] .
\end{aligned}
$$

In the particular case when $\mu_{f_{0}}=\mu_{f_{R}}=0$, one recovers Eq. (11).

\section{APPENDIX D: SIMULATIONS}

The aim of the simulations is to provide a numerical test of the main results in Secs. III A, IV A, and V A. We wrote a MATLAB [31] script that integrates $N=10^{5}$ trajectories of a particle subject to random collisions and computes numerical estimates $m_{\chi}(t)$ of $\mu_{\chi}(t)$ and $s_{\chi}^{2}(t)$ of $\sigma_{\chi}^{2}(t)$. We chose an algorithm based on discrete time steps (as discussed below) due to its simplicity, easiness of implementation and acceptable performance, with execution times well suited for execution on a single, one core, computer. We did not pursue other approaches, although other more efficient alternatives may exist.

We start by setting the initial conditions. We randomly generate $N$ positions $\left\{x_{1}(0), x_{2}(0), \ldots, x_{n}(0), \ldots, x_{N}(0)\right\}$ distributed $g_{0}(x)$, and $N$ random velocities $\left\{v_{1}(0), v_{2}(0), \ldots, v_{n}(0), \ldots, v_{N}(0)\right\}$ distributed $f_{0}(v)$. We then advance the dynamics in all trajectories by a time step $\Delta t=\left(10^{3} \gamma\right)^{-1}$. This choice is made so that $\Delta t \ll \gamma^{-1}$ and then, as discussed in Appendix A, the probability of undergoing one collision during the time interval $\Delta t$ is $\gamma \Delta t \ll 1$, a constant value (since $\gamma>0$ is fixed). The probability of having more than one collision is negligible. Thus, collisions are overall distributed Poisson $(\gamma t)$ as shown in Fig. 12.

For each trajectory $n$, location changes by an amount $v_{n}(0) \Delta t$ so that $x_{n}(\Delta t)=x_{n}(0)+v_{n}(0) \Delta t$. If no collisions happen in the trajectory during the time interval $\Delta t$, then there is no change in velocity and $v_{n}(\Delta t)=v_{n}(0)$. Otherwise, if a collision happens, then a new random velocity is generated which replaces the original velocity. For example, in the case of Sec. III B, we make $v_{n}(\Delta t)=\mathcal{V}_{n}$ with $\mathcal{V}_{n} \sim f_{R}(v)$.

The algorithm continues in a similar fashion for later time steps, so that for trajectory $n$ we have

$$
\begin{aligned}
& x_{n}(t+\Delta t)=x_{n}(t)+v_{n}(t) \Delta t, \\
& v_{n}(t+\Delta t)= \begin{cases}v_{n}(t) & \text { if no collision happens, } \\
\mathcal{V}_{n} & \text { if there is a collision. }\end{cases}
\end{aligned}
$$

In this expression it is understood that $t=j \Delta t$ for some integer $j \geqslant 1$.

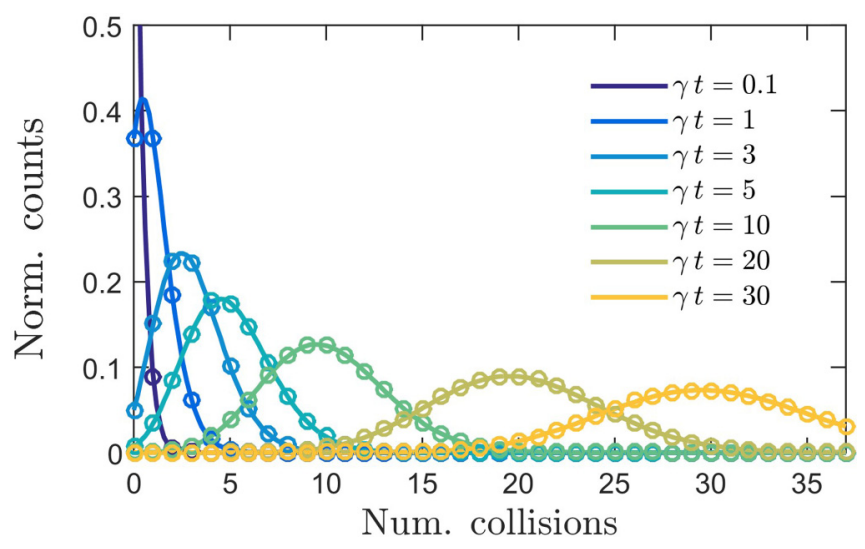

FIG. 12. Distribution of number of collisions. The circles are numerically computed as the number of trajectories having undergone a given number of collisions (horizontal axis) by time $t$, divided by the total number of trajectories $N=10^{5}$. Different colors correspond to different $t$ using a fixed $\gamma=1$. The solid lines are plots of the Poisson $(\gamma t)$ distribution. There is good agreement between the simulations and the expected collision probabilities.

To simulate the occurrence of collisions, we generate in each time step $N$ random numbers $\theta_{n}$ (one for each trajectory) distributed Uniform in the interval $[0,1]$. The probability that $\theta_{n} \leqslant \gamma \Delta t$ is equivalent to the probability of undergoing a collision during $\Delta t$. Therefore, trajectory $n$ will be taken as experiencing a collision if $\theta_{n} \leqslant \gamma \Delta t$.

The walker location statistics are then computed by taking the sample mean and sample variance [28] of $\left\{x_{1}(t), \ldots, x_{n}(t), \ldots, x_{N}(t)\right\}$,

$$
\begin{aligned}
m_{\chi}(t) & =\frac{1}{N} \sum_{n=1}^{N} x_{n}(t), \\
s_{\chi}^{2}(t) & =\frac{1}{N-1} \sum_{n=1}^{N}\left[x_{n}(t)-m_{\chi}(t)\right]^{2} .
\end{aligned}
$$

The transport coefficient is determined from the definition [Eq. (13)] by numerically computing the derivative using these sample statistics.

The finite sample size means that results will suffer from statistical errors. These are especially important at large $t$ due to the spreading of the particles and larger variances in walker location. Furthermore, the step size can affect the overall distribution of the collisions (the Poisson distribution is a limiting case for short $\Delta t$; see Sec. II), and add an uncertainty to the distance traveled by the walker within a time step. The latter is due to the fact that a collision only changes the velocity at the end of the step instead of at any moment. This also makes the algorithm artificially set the collision probability to zero at very early times of the walk $(t \lesssim \Delta t)$. Thus, there is an inherent limit on the minimum resolution achievable at early times and we expect simulations to be valid only when $t>\Delta t$.

The choices $N=10^{5}, \Delta t=\left(10^{3} \gamma\right)^{-1}$, give consistent results for the typical collision rates and velocities used in this paper, as benchmarked against larger samples and other choices of time steps. 


\section{APPENDIX E: RELATIONSHIP BETWEEN ADDITIVE VELOCITY JUMPS AND DIFFUSION OF VELOCITIES}

In the case of additive velocity jumps we have $f(u, v)=$ $f_{A}(u-v)$, which upon insertion in Eq. (2) yields

$$
\left(\frac{\partial}{\partial t}+v \frac{\partial}{\partial x}+\gamma\right) p(x, v, t)=\gamma \int f_{A}(u-v) p(x, u, t) d u \text {. }
$$

For what follows, we assume the following functional form for $f_{A}$ :

$$
f_{A}(v)=\frac{1}{2}\left[\delta\left(v-\Delta_{v}\right)+\delta\left(v+\Delta_{v}\right)\right],
$$

where $\Delta_{v}>0$ is a fixed parameter which is small (we shall be more precise on what this means below). This PDF models a simple situation in which, upon a collision, the velocity of the walker increases or decreases (with equal probability) by a fixed amount $\Delta_{v}$.

Replacing into the RHS of Eq. (E1) yields

$$
\begin{aligned}
& \int f_{A}(u-v) p(x, u, t) d u \\
& =\frac{1}{2} \int\left[\delta\left(u-v-\Delta_{v}\right)+\delta\left(u-v+\Delta_{v}\right)\right] p(x, u, t) d u
\end{aligned}
$$

$$
\begin{aligned}
& =\frac{1}{2}\left[p\left(x, v+\Delta_{v}, t\right)+p\left(x, v-\Delta_{v}, t\right)\right] \\
& =\frac{1}{2}\left[2 p(x, v, t)+\Delta_{v}^{2} \frac{\partial^{2}}{\partial v^{2}} p(x, v, t)+O\left(\Delta_{v}^{4}\right)\right] \\
& \approx p(x, v, t)+\frac{\Delta_{v}^{2}}{2} \frac{\partial^{2}}{\partial v^{2}} p(x, v, t),
\end{aligned}
$$

where we have performed a Taylor expansion in $v$ around $p(x, v, t)$ and neglected terms of $O\left(\Delta_{v}^{4}\right)$. The smallness of $\Delta_{v}$ is then the condition that this expansion holds.

Equation (E1) for this particular case then becomes

$$
\left(\frac{\partial}{\partial t}+v \frac{\partial}{\partial x}\right) p(x, v, t)=\left(\frac{\gamma \Delta_{v}^{2}}{2}\right) \frac{\partial^{2}}{\partial v^{2}} p(x, v, t),
$$

which shows a diffusive term for the velocities.

The function $f_{A}$ need not be constrained to having "small" jumps but can also model macroscopic velocity changes. It also can have nonzero mean or a skewed profile. The theory in Sec. IV A can treat all these situations in full generality.
[1] R. Metzler and J. Klafter, The random walk's guide to anomalous diffusion: A fractional dynamics approach, Phys. Rep. 339, 1 (2000).

[2] V. Zaburdaev, S. Denisov, and J. Klafter, Lévy walks, Rev. Mod. Phys. 87, 483 (2015).

[3] A. Bovet, Suprathermal ion transport in turbulent magnetized plasmas, Ph.D. dissertation, École Polytechnique Fédérale de Lausanne, Switzerland, 2015.

[4] R. Gorenflo, A. Vivoli, and F. Mainardi, Discrete and continuous random walk models for space-time fractional diffusion, Nonlin. Dyn. 38, 101 (2004).

[5] J. Blum, S. Bruns, D. Rademacher, A. Voss, B. Willenberg, and M. Krause, Measurement of the Translational and Rotational Brownian Motion of Individual Particles in a Rarefied Gas, Phys. Rev. Lett. 97, 230601 (2006).

[6] R. Huang, I. Chavez, K. M. Taute, B. Lukic, S. Jeney, M. G. Raizen, and E.-L. Florin, Direct observation of the full transition from ballistic to diffusive Brownian motion in a liquid, Nat. Phys. 7, 576 (2011).

[7] H. G. Othmer, S. R. Dunbar, and W. Alt, Models of dispersal in biological systems, J. Math. Biol. 26, 263 (1988).

[8] E. A. Codling, M. J. Plank, and S. Benhamou, Random walk models in biology, J. R. Soc. Interface 5, 813 (2008).

[9] G. H. Weiss, Some applications of persistent random walks and the telegrapher's equation, Physica A 311, 381 (2002).

[10] S. Godoy, L. S. Garcia-Colin, and V. Micenmacher, Generalized Landauer equation: Absorption-controlled diffusion processes, Phys. Rev. E 59, 6180 (1999).

[11] G. E. Uhlenbeck and L. S. Ornstein, On the theory of Brownian motion, Phys. Rev. 36, 823 (1930).

[12] J. L. Doob, The Brownian movement and stochastic equations, Ann. Math. 43, 351 (1942).
[13] J. Masoliver, K. Lindenberg, and G. H. Weiss, A continuoustime generalization of the persistent random walk, Physica A 157, 891 (1989).

[14] J. Masoliver and K. Lindenberg, Continuous time persistent random walk: A review and some generalizations, Eur. Phys. J. B 90, 107 (2017).

[15] G. H. Weiss, Aspects and Applications of the Random Walk, North Holland (Elsevier Science, Amsterdam, 1994).

[16] V. Zaburdaev, M. Schmiedeberg, and H. Stark, Random walks with random velocities, Phys. Rev. E 78, 011119 (2008).

[17] G. H. Weiss, J. Masoliver, K. Lindenberg, and B. J. West, Firstpassage times for non-Markovian processes: Multivalued noise, Phys. Rev. A 36, 1435 (1987).

[18] J. Masoliver, Three-dimensional telegrapher's equation and its fractional generalization, Phys. Rev. E 96, 022101 (2017).

[19] J. Duplat, S. Kheifets, T. Li, M. G. Raizen, and E. Villermaux, Superdiffusive trajectories in Brownian motion, Phys. Rev. E 87, 020105(R) (2013).

[20] R. R. Coveyou, R. R. Bate, and R. K. Osborn, Effect of moderator temperature upon neutron flux in infinite, capturing medium, J. Nuclear Energy 2, 153 (1956).

[21] J. Wesson, Tokamaks, 3rd ed. (Oxford University Press, Oxford, 2004).

[22] I. Furno, F. Avino, A. Bovet, A. Diallo, A. Fasoli, K. Gustafson, D. Iraji, B. Labit, J. Loizu, Müller, G. Plyushchev, M. Podesta, F. M. Poli, P. Ricci, and C. Theiler, Plasma turbulence, suprathermal ion dynamics and code validation on the basic plasma physics device TORPEX, J. Plasma Phys. 81, 345810301 (2015).

[23] A. Bovet, A. Fasoli, P. Ricci, I. Furno, and K. Gustafson, Nondiffusive transport regimes for suprathermal ions in turbulent plasmas, Phys. Rev. E 91, 041101(R) (2015). 
[24] S. Servidio, C. T. Haynes, W. H. Matthaeus, D. Burgess, V. Carbone, and P. Veltri, Explosive Particle Dispersion in Plasma Turbulence, Phys. Rev. Lett. 117, 095101 (2016).

[25] L. F. Richardson, Atmospheric diffusion shown on a distanceneighbour graph, Proc. R. Soc. London A 110, 709 (1926).

[26] B. Liebchen, R. Büchner, C. Petri, F. K. Diakonos, F. Lenz, and P. Schmelcher, Phase space interpretation of exponential Fermi acceleration, New J. Phys. 13, 093039 (2011).

[27] J. J. Duderstadt and W. R. Martin, Transport Theory (John Wiley and Sons, New York, 1979).

[28] A. Gut, Probability: A Graduate Course, 2nd ed. (Springer, New York, 2013).
[29] R. N. Bracewell, The Fourier Transform and Its Applications, 3rd ed. (McGraw-Hill, New York, 2000), pp. 5-6.

[30] G. B. Arfken, F. E. Harris, and H.-J. Weber, Mathematical Methods for Physicists: A Comprehensive Guide, 7th ed. (Academic Press, Waltham MA, 2012), pp. 75-79, 985-987.

[31] See https://www.mathworks.com/products/matlab.html for a description of the MATLAB software.

[32] M. Kac, A stochastic model related to the telegrapher's equation, Rocky Mountain J. Math. 4, 497 (1974).

[33] S. Ross, Introduction to Probability Models, 9th ed. (Elsevier, Burlington MA, 2007), pp. 119-120.

[34] See https://www.wolfram.com/mathematica/ for a description of the Mathematica software, v. 10.1.0.0. 